\title{
The $\operatorname{Ag}_{x} \mathrm{Si}_{y}(x=1-5, y=1-5, x+y=5)$ Clusters
}

\author{
Mariana Virginia Popa ${ }^{1,2}$ \\ ${ }^{1}$ Electronic and Telecomunication, Autonomous University of the Hidalgo State, Mexico \\ ${ }^{2}$ Regularización 2000, C/Dr. Agustin Torres Cravioto 102B, col. Doctores, Pachuca, Hidalgo, Mexico
}

Email address:

virginia_popa@yahoo.com.mx,pmarianavirginia@yahoo.com

\section{To cite this article:}

Mariana Virginia Popa. The $\mathrm{Ag}_{\mathrm{x}} \mathrm{Si}_{\mathrm{y}}(\mathrm{x}=1-5, \mathrm{y}=1-5, \mathrm{x}+\mathrm{y}=5)$ Clusters. International Journal of Computational and Theoretical Chemistry. Special Issue: Electronic Proprieties in Computational Chemistry. Vol. 3, No. 3-1, 2015, pp. 19-35. doi: 10.11648/j.ijctc.s.2015030301.12

\begin{abstract}
En this paper are presented the theorics work for clarify the structure of all cluster which levels of theory describe better the propriety of the $\mathrm{Ag}_{\mathrm{x}} \mathrm{Si}_{\mathrm{y}}$ cluster. For $\mathrm{Ag}_{\mathrm{x}} \mathrm{Si}_{\mathrm{y}}$ are reach different value of the bond, ionization potentials and frequencies, electron affinities and biding energy method employed ab initio and relativystic bases. Are optimization with PBE/LANL2DZ level of theorie. I employed relativistic LANL2DZ bases in this work and PBE functional of exchenge and coreletion PerdewBurke-Ernzerhof who employed the GCA(Genaralizad Gradient Approximation), this are better for the energy, and different energy structurale.
\end{abstract}

Keywords: $\mathrm{Ag}_{\mathrm{x}} \mathrm{Si}_{\mathrm{y}}$ clusters, Electronic Proprieties, Biding Energy, Electron Affinity and Ionization Potentials

\section{Introduction}

Many series of elemental clusters have been characterized in detail with a variety of different levels of theorie and experimental techniques. Very little is known about the structure $\mathrm{A}_{\mathrm{x}} \mathrm{B}_{\mathrm{y}}$ of clusters in general. Elucidating of this structure is a challenging problem that requires piecing together informations from different experiments and theory. The study of $\mathrm{Ag}_{\mathrm{x}} \mathrm{Si}_{\mathrm{y}}$ is important for the practical importance in the new electronic materials. Are mencioned in the papers the structure and another proprieties of silver and silicon clusters. The pople are now the difference of the clusters and bulk-state. Ideas like "super-atoms", "magic numbers" or "fission" in clusters provoques a wider class of scientists to study this "relatively" new area of the phisical sciences. Growing interest in the stabilities of small clusters and the evolution of bulk properties from cluster proprieties is also due to the emergence of new science.

$\mathrm{Ab}$ initio effective core potentials (ECPs) have been generated to replace the Coulomb, echenge, and coreorthogonality effects of the chemically inert core electron in the transition metal atoms.

In LANL2DZ the relativistic mass-velocity and Darwin effect on the valence electrons are incorporated in the relativistic ECP's generated for the havier $(\mathrm{Z}>36)$ elements.

The "core" ns and np orbitals do not play any direct role in the chemical bonding in this species, since their binding energies are much larger than electrons in the valence
shell.The core electrons: $\operatorname{Ag}[$ core $] 4 s^{2} 4 p^{6} 4 d^{10} 5 s^{1}$. The calculations are with the program Gaussian 03 .

In this studie are important now the influence of delectrons at the outer orbitals, overlaped by the partially filled s-orbitals, which makes the studiy of silver clusters interesting and challenging. The dissociation energies for the loss of an atom showed strong odd-even alternation. The electronic properties of silver cluster and the stability of the clusters are strongly dominated by the filled d electrons screening the oscillator strenght of valence electrons but are not know for $\mathrm{Ag}_{\mathrm{x}} \mathrm{Si}_{\mathrm{y}}$. The majority of spectroscopic phenomena can be explained in terms of $\mathrm{s}$ electrons. Topologically different clusters and clusters belonging to different symmetry groups have been identified and studied in detail for $\mathrm{Ag}_{\mathrm{x}} \mathrm{Si}_{\mathrm{y}}$ clusters. In the potential energy surface (PES) the local minimum, meaning that it is the lowest point in some limited region of the potential surface, or it can be the global minimum, the lowest energy point anywhere on the potential surface. Different minima corresponding to different conformations or stuctural isomers in the case of single molecules. A point which is a maximum in one direction and a minimumin the other is called a saddle point. A saddle point corresponds to a transition structure connecting the two equilibrium structure. A point on the potential energy surface where the force are zero is called a stationary point. By definition, a structure which has $n$ imaginary frequencies is an $\mathrm{n}^{\text {th }}$ order saddle point. In this work are not reported all transition structure with imaginary 
frequencies that are important for path of reaction between the minima local point in the PES. Are studie all proprieties because are important for the chemical reaction when are posible form secundary product because this structure are more energy and exist for little timp for transformation in another structure or reacting.

\section{Results and Discussion}

In this work full geometry optimizations have been carried out and are reported only the must stable structure with the positive frecuencies values.

The local density approximation (LDA) in densityfunctional theory (DFT) has been used successfully to calculate the structural properties of solids, including the calculations of the stability, lattice constant, bulk moduli, and phonon spectra, for the last two decades, for this motive I used in this paper PBE theory.

The overestimation of binding energy of the molecule or solids and the underestimation of the gap in insulators and bond lengths are well known deficiencies of the LDA which limits its further application. Computationally, it is simple to extend the LDA functional to the GGA functional with simple gradient calculations of the density at minimal cost.

Ortiz and Ballone applied the earlier version of the GGA functional to atoms, molecules, and solids and characterized the basic properties of the GGA functional [1, 2]. In this paper we consider the simplified version of the GGA functional by Perdew, Burke, and Ernzerhof (PBE)[3] which satisfies many exact properties of DFT. I employed $\mathrm{SCF}=\mathrm{QC}$, quadratically convergent SCF procedure [4], for optimization in Gaussian for all structure neutral, anions and cations.

Acording to molecular orbital (MO) theory the ionization potential of a molecule is simple the orbital energy of the HOMO and the electron affinity is the orbital energy of the LUMO, with change in sign:

$$
\begin{aligned}
& \mathrm{I}=-\varepsilon_{\text {HOMO }} \\
& \mathrm{A}=-\varepsilon_{\text {LUMO }}
\end{aligned}
$$

These are consequences of Koopmans theorem.

\section{Distance in Clusters}

\subsection{Neutral Dimers, Neutral Anions and Cations}

b)

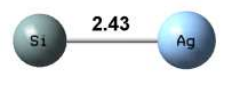

C1, -4070.037 c)

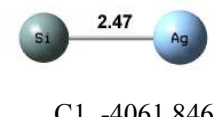

Fig. 1. The structure of dimer clusters with PBE/LANL2DZ: a) neutral; b) anions; c) cations. Are reported symmetrie and electronic state. The energy of clusters in $\mathrm{eV}$.

Are observed that the anion and cation cluster are minor distance that the neutral cluster. The cluster Fig. 1 a) are the $\alpha$ orbital HOMO with -0.163 energy and $\beta$ orbital HOMO with -0.143 energy, degenerate; the LUMO orbital $\alpha$ are -0.158 energy and $\beta$ orbital LUMO with -0.111 energy. The HOMO for cation cluster are 0.057 for $\alpha$ and $\beta$ and LUMO 0.037 energy. For anion cluster -0.415 for HOMO and -0.386 energy for LUMO. For this degenerate orbital the distance in neutral cluster are minor that cation and anion cluster. In literatura for silver cluster are not reported this tip of distance and variation of distance beteen neutral, anion and cation cluster [5].

\subsection{Neutral Trimer, Neutral, Anions and Cations}

For the structure in Fig. 2 a) I the HOMO are -0.114 and LUMO -0.133 energy, for 2 a) II -0.151 and -0.193 hartree, for 2 a) III -0.178 hartree and -0.150 hartree and 2 a) IV the
HOMO are -0.187 and LUMO are -0.113 hartree. In this cluster are present the Jahn -Teller effect, for Fig. 2 b) I when $\alpha$ HOMO are 0.019 hartree and $\beta$ HOMO are 0.000 hartree, the LUMO are 0.061 and 0.057 hartree. In Fig. 2 b) II the HOMO are 0.021 and LUMO 0.010. In the Fig. 2 b) III the cluster for $\alpha$ HOMO are 0.040 and $\beta$ HOMO 0.048 hartree, for LUMO $\alpha$ and $\beta 0.007$ and 0.055 hartree. In Fig.2 c) I $-0.360 \alpha$ HOMO and $-0.326 \beta$ HOMO, for LUMO are 0.340 and -0.319 hartree. In Fig. 2 c) II $-0.380 \alpha$ HOMO and $-0.350 \beta$ HOMO, for LUMO are -0.351 and -0.340 hartree. In Fig. 2 c) III HOMO -0.343 and LUMO -0.352 hartree and Fig. 2 c) IV for HOMO -0.380 and LUMO -0.387 hartree. In Fig. $2 \mathrm{c}$ I the obtuse triangle are more distance that in Fig. $2 \mathrm{~b}$ II for the Jahn Teller effect. The structure in Fig. 2 a) I are the same distance with the Fig. 2 b) III for anion cluster. For neutral structure the isoscele Fig. 2 a) III are minor energy, for anion the linear structure and cation the obtuse triangle. a) I

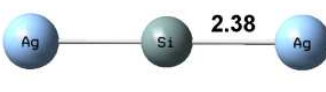

C1, 1SG, -8034.865
II

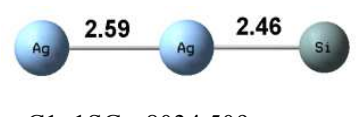

C1, 1SG, -8034.509
III

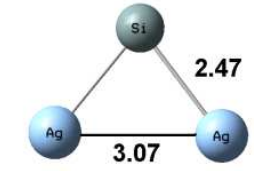

C2, 1A1, -8036.588
IV

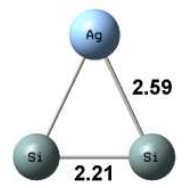

C2, 2B1, -4175.469 
b) I

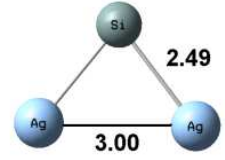

C2, 2B1, -4177.553

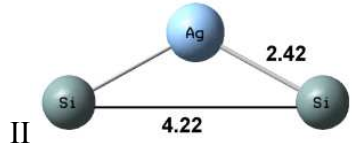

C2, 1A1, -4174.329
III

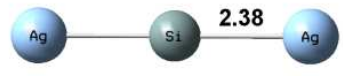

C1, -8036.379 c) I

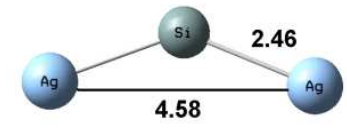

C2 , 2A1, -8029.49979

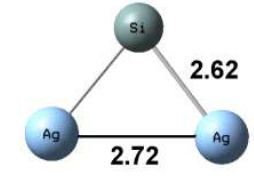

C2, 2B2, -8029.401

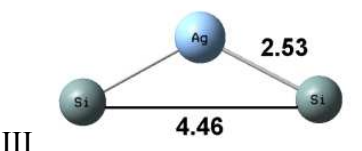

C2, 1A1, -4165.302
IV

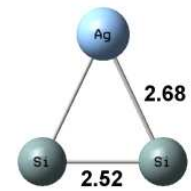

C2, 1A1, -4167.276

Fig. 2. The structure of trimer clusters with PBE/LANL2DZ,: a) neutral; b) anions; c) cations. Are reported symmetrie and electronic state. The energy of clusters in $\mathrm{eV}$.

\subsection{Tetramer, Neutral, Anions and Cations}

In the Fig. 3a) I the HOMO are -0.157 hartree and LUMO 0.179 hartree, Fig. 3a) II the HOMO are -0154 hartree and LUMO -0.169 hartree. In Fig. 3 a) III the $\alpha$ HOMO are -0.184 and $\beta$ HOMO are -0.178 hartree, for LUMO are -0.173 and 0.172 hartree. In Fig. 2 a) IV the HOMO are -0.161 and LUMO -0.221 hartree. In Fig. 3a) $\mathrm{V}$ the $\alpha$ HOMO are -0.161 and $\beta$ HOMO are -0.133 hartree, for LUMO are -0.104 and 0.097 hartree, are degenerate orbital. In the Fig. 3 b) I the HOMO are 0.022 and LUMO -0.002 hartree, Fig. 3 b) II for anion cluster $\alpha$ HOMO occuped with one electron are -0.029 and $\beta$ HOMO are -0.027 , for $\alpha$ LUMO 0.035 and $\beta$ LUMO are 0.056 hartree. In Fig. 3 b) III the HOMO are 0.023 and LUMO -0.005 hartree, in Fig. 3 b) IV the HOMO are -0.022 and LUMO are -0.013 hartree. In Fig. 3 b) V the HOMO are 0.007 and LUMO 0.00 Hartree, in Fig. 3 b) VI the HOMO are -0.022 and LUMO are 0.041 hartree. In Fig. 3 b) VIII the HOMO are -0.013 and LUMO -0.009 , in Fig. 3 b IX the HOMO are -0.008 the LUMO are 0.008 hartree, the 2 electron with oposite spin are occuped the same orbital. The Fig. $3 \mathrm{~b}) \mathrm{X}$ the $\alpha \mathrm{HOMO}$ are -0.019 and $\beta$ HOMO are -0.026 , but the $\alpha$ LUMO are positive values 0.039 and $\beta$ LUMO are 0.048 . The Fig. 3 c) I are cation cluster when HOMO are -0.331 and LUMO are -0.348 , in Fig. 3 c) II $\alpha$ HOMO are -0.312 and $\beta$ HOMO -0.379 , for $\alpha$ LUMO are -0.390 and $\beta$ LUMO are -0.361 hartree. In Fig. 3 c) III the HOMO occuped with 2 electrons are -0.316 energy and LUMO are -0.362 hartree, Fig. 3 c) IV for HOMO are -0.375 and for LUMO are -0.324 hartree, Fig. 3 c) V for HOMO are 0.345 and LUMO -0.383 hartree, Fig. 4 c) VI for HOMO are 0.378 and for LUMO are -0.337 hartree and Fig. 3 c) VII for $\alpha$ HOMO are -0.348 and $\beta$ HOMO are -0.366 hartree. In Fig. c) II and Fig. b) II are view that in anion cluster are more greater the distance between Si-Si. Compared Fig. 3 c) VII, b) X and 3 a) IV the anion are more greater distance beteen the atoms in this sens are covalent simple biding more that another structure. The structure $3 \mathrm{~b}$ ) III and $3 \mathrm{~b}$ ) IX are the $1 / 2 \pi$ covalent biding, for this motive in both structure the distance are minor that the simple covalent biding. a) I

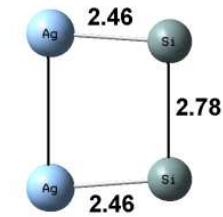

C2,1A1, -8140.277
II

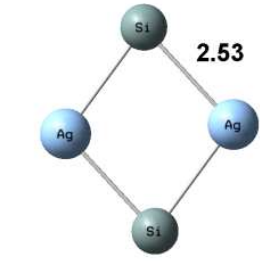

D2, $1 \mathrm{AG},-8140.564$

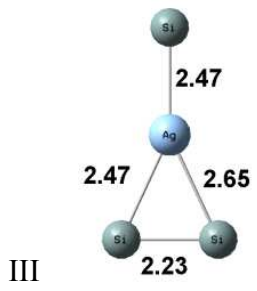

C1 ,1A2, -4278.71129

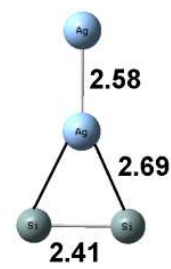

IV

C2, 1B2, -8140.06134

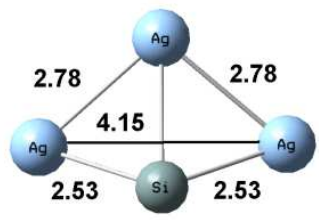

C1, 2A, -12003.755 b)

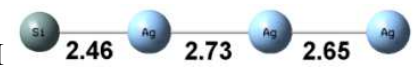

C1, 1SG, -12003.056

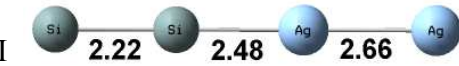

C1, -8143.74489 


\section{III}

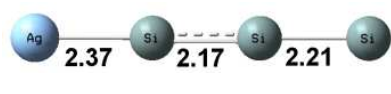

C1, -4281.198

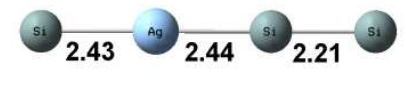

C1, -4281.073

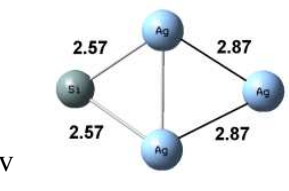

C2, 1A1, -12004.486

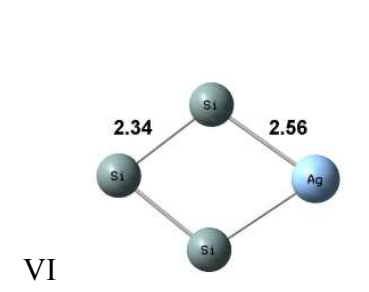

$\mathrm{C} 2,1 \mathrm{~A} 1,-4283.742$

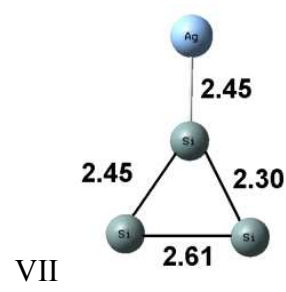

C1, 1A1, -4283.137

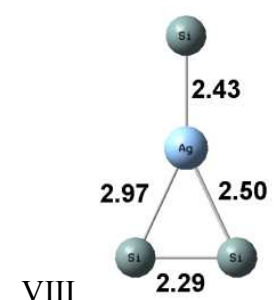

C1, 1A1, -4281.173

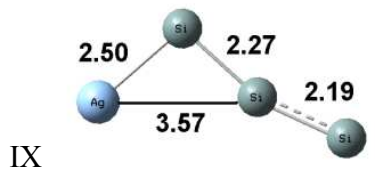

C1, 1A', -4282.778

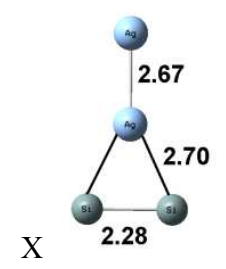

C2, 2B1, -4282.77841

c)
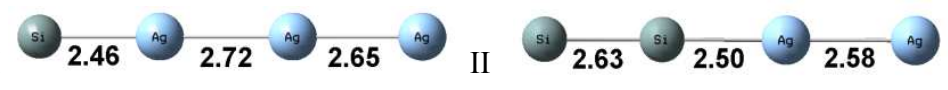

C1, -11994.639

C1, -8131.954

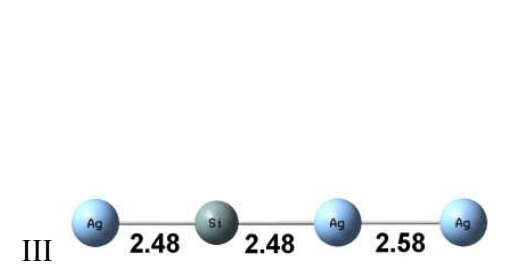

C1,1SG, -11994.724

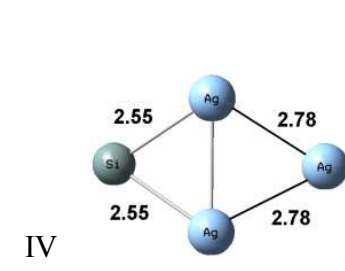

$\mathrm{C} 2,1 \mathrm{~A} 1,-11997.233$

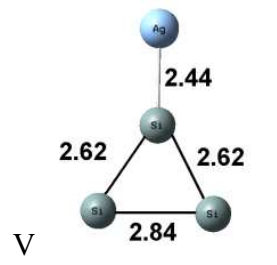

$\mathrm{C} 2,1 \mathrm{~A} 1,-4271.353$

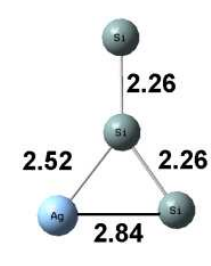

$\mathrm{C} 1,1 \mathrm{~A}^{\prime},-4273.657$

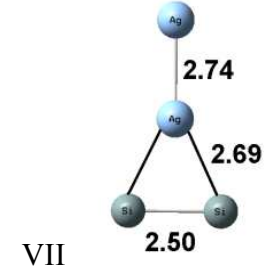

$\mathrm{C} 2,1 \mathrm{~A} 1,-8133.405$

Fig. 3. The structure of tetramer clusters with PBE/LANL2DZ: a) neutral; b) anions; c) cations. Are reported symmetrie and electronic state. The energy of clusters in $\mathrm{eV}$.

\subsection{Pentamer, Neutral, Anions and Cations}

In Fig. 4 a) I the $\alpha$ HOMO are -0.172 and $\beta$ HOMO are 0.163 hartree, for $\alpha$ LUMO are -0.158 and $\beta$ LUMO -0.156 hartree, Fig. 4 a) II the $\alpha$ HOMO are -0.175 and $\beta$ HOMO are -0.165 hartree and for $\alpha$ LUMO are $-0.171-0.155$ and $\beta$ LUMO, Fig. 3 a) III the $\alpha$ HOMO are -0.201 and $\beta$ HOMO are -0.196 for $\alpha$ LUMO are -0.200 and $\beta$ LUMO -0.185 . In Fig. 4 a) IV the $\alpha$ HOMO are -0.177 and $\beta$ HOMO are -0.150 hartree for $\alpha$ LUMO are -0.148 and $\beta$ LUMO -0.144, Fig. 4 a) $\mathrm{V}$ for $\alpha$ HOMO are -0.167 and $\beta$ HOMO are -0.138 , for $\alpha$ LUMO are -0.139 and $\beta$ LUMO are -0.136 . For Fig. 4 a) VI for HOMO are -0.187 hartree and LUMO -0.146 , Fig. 4 a) VII for HOMO are -0.192 and LUMO -0.145. For Fig. 4 a) VIII for $\alpha$ HOMO are -0.149 and $\beta$ HOMO are -0.129 , for $\alpha$ LUMO are -0.116 and for $\beta$ LUMO are -0.113 . For Fig. 4 a) IX for $\alpha$ HOMO are -0.149 and $-\beta$ HOMO are 0.129 , for $\alpha$ LUMO are -0.116 and $\beta$ LUMO are -0.113 . For Fig. 4 a) $\mathrm{X}$ for $\alpha$ HOMO are -0.177 and $\beta$ HOMO are -0.151 , for $\alpha$ LUMO are -0.148 and $\beta$ LUMO are -0.144 . For Fig. 4 b) I the HOMO are -0.022 and LUMO are -0.029 , in Fig. 4 b) II $\alpha$ HOMO are -0.201 and $\beta$ HOMO are -0.196 , for $\alpha$ LUMO are -0.200 and $\beta$ LUMO are -0.185 . In Fig. 4 b) III the HOMO are -0.011 and LUMO are 0.023 , in Fig. 4 b) IV the $\alpha$ HOMO are -0.005 and $\beta$ HOMO are 0.019 , for $\alpha$ LUMO are 0.031 and $\beta$ LUMO are 0.038 . In Fig. 4 c) I are -0.350 hartree for HOMO and -0.342 hartree for LUMO, in Fig. 4 c) II the $\alpha$ HOMO are -0.338 and $\beta$ LUMO are -0.312 , but $\alpha$ LUMO are -0.317 and $\beta$ LUMO are -0.308 , in Fig. 4 c) III the $\alpha$ HOMO are -0.337 and $\beta$ HOMO are -0.310 , the $\alpha$ LUMO are -0.313 and $\beta$ LUMO are -0.306 , in Fig. 4 c) IV the HOMO are 0.360 and LUMO -0.264 . In the Fig. 4 a) I and II and b) I are un $2 \frac{1}{2} \pi$ biding and one double biding with minor distance that single biding covalent. For this reason this cluster are transformed in another cluster or reaction very quick. a) I

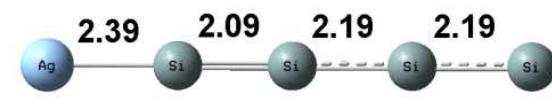

C1, -4384.841

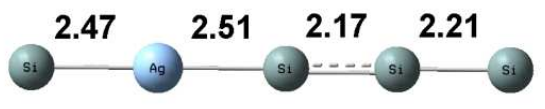

C1, -4383.969 

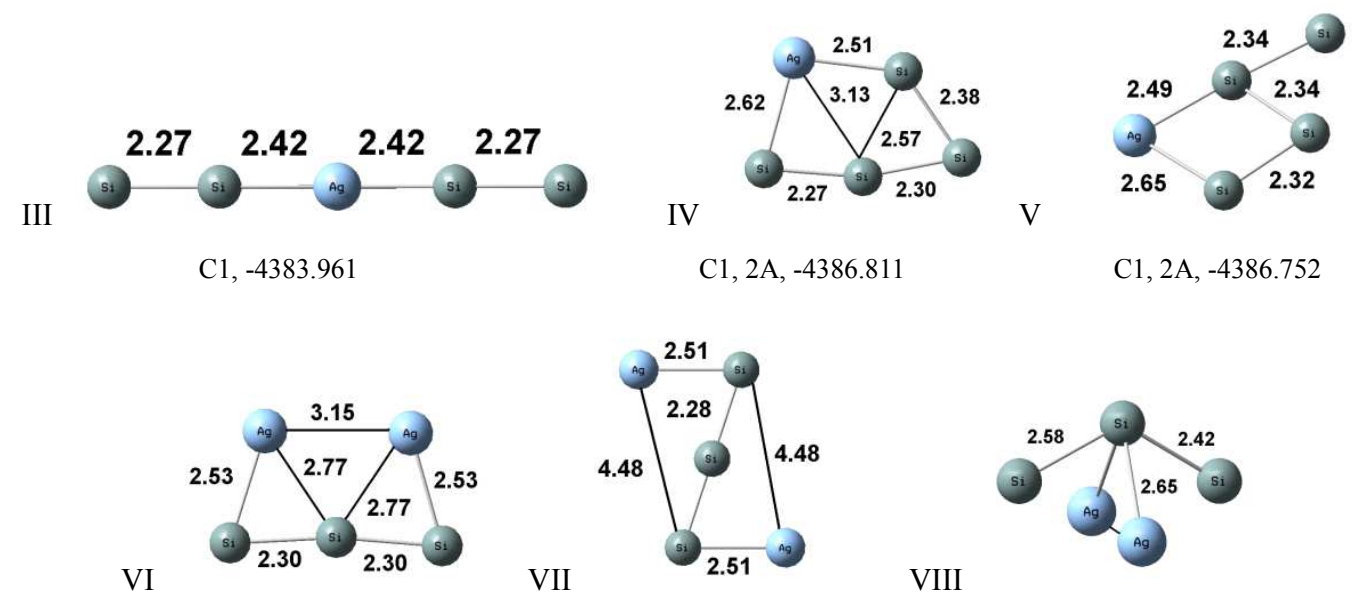

C1, 1A, -4386.811

VII

C1, A1, -8247.923

VIII

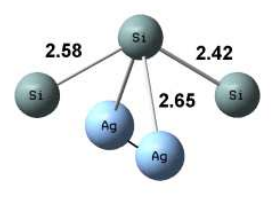

C1, 2A, -12109.291

IX

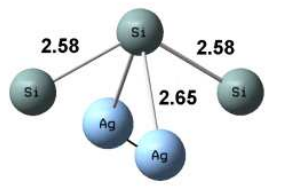

C1, 2A, -12109.291
X

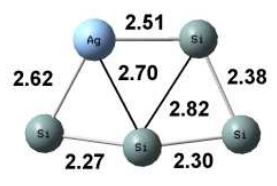

C1, 2A, -4386.811

C1, 2A, -4386.752

b) I
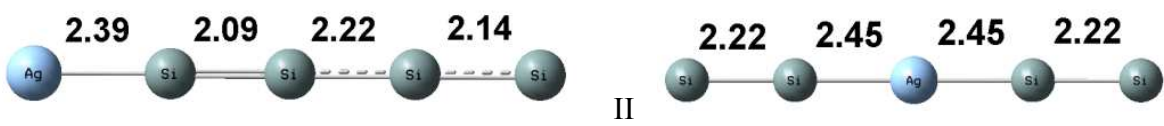

C1, 1SG, -161.233029

C1, -161.1075451

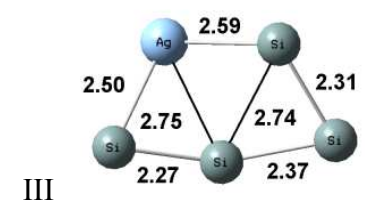

C1, 1A, -161.2963853

IV

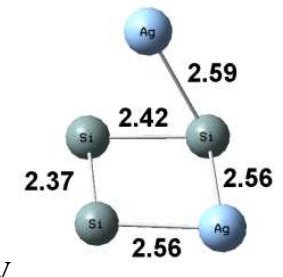

C1, 2A, -303.1860191

c) I

\section{$\begin{array}{llll}2.35 & 2.42 & 2.42 & 2.35\end{array}$}

C1, 1SG, -4376.545

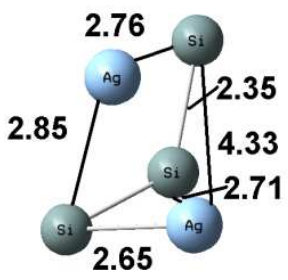

C1, 2A, -8241.041

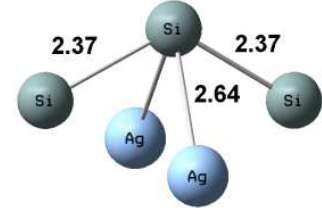

III

C1, 2A, -8241.108

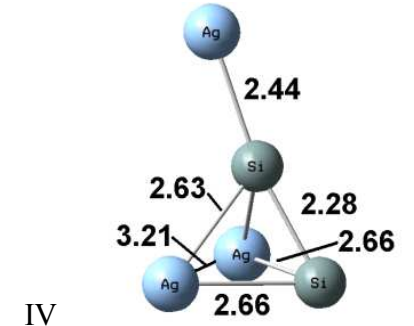

C1, A1, -12103.624

Fig. 4. The structure of pentamer clusters with PBE/LANL2DZ: a) neutral; b) anions; c) cations. Are reported symmetrie and electronic state. The energy of clusters in $\mathrm{eV}$.

\section{Biding Energy for Atom}

Is important to study the biding energy for atom because to refer at stability of clusters. Are graphical the value of the biding energy of the neutral cluster, anions and cations, Fig. 5-
7.

Calculate with $\frac{E_{b}}{n}=\frac{x E_{x}+y E_{y}+\cdots+m E_{m}-E_{n}}{n}$, where $\mathrm{E}_{\mathrm{n}}$ are the total energy for the neutral species optimizated with $\mathrm{n}$ atoms $\left(\mathrm{Ag}_{\mathrm{x}} \mathrm{Si}_{\mathrm{y}}\right.$ in this work); $\frac{E_{b}^{ \pm}}{n}=\frac{x E_{x}+y E_{y}+\cdots+m E_{m}-E_{n}^{ \pm}}{n}$, where $\mathrm{n}$ are 
the number of the total atoms in the cluster, $\mathrm{x}$ are the number of the atom of element $\mathrm{x}$ ( $\mathrm{Ag}$ in this work) $\mathrm{y}$ are the number of atom for the another element ( $\mathrm{Si}$ in this work), the $\mathrm{E}_{\mathrm{x}}$ are the energy of the neutral element $x$ and $E_{y}$ are the energy of the neutral element $\mathrm{y}, \mathrm{m}$ are the number of atom of element $\mathrm{m}$ and $\mathrm{E}_{\mathrm{m}}$ are the energy of the neutral element $\mathrm{m}$, and $E_{n}^{ \pm}$are the energy of the anion or cation cluster $\left(\mathrm{Ag}_{\mathrm{x}} \mathrm{Si}_{\mathrm{y}}\right.$ in this work). The biding energy of anion per atom for the anion and cation cluster are employed with this ecuation because the cluster are charge negative or positive with the sum of the charge of all atoms in the cluster. In Fig. 5 are observed the binding energy per atom for neutral structure, Fig 2a) IV, are greater $2.77 \mathrm{eV}$ that another cluster with 3 atoms and 4 atoms. For 5 atoms the binding anergy are grater in comparation with 2, 3 and 4 atoms. For Fig 4a) I with the frecuencies negative are $3.15 \mathrm{eV}$ and are greater that another linear stucture 4a) III and the trapezoidal form are the same energy with the another structure. The linear form Fig. 3a) IV are little binding energy 2.04 near with the structure with 2 atoms, Fig. 6 . The form T for cluster with 4 atoms are very near energy with the structure with 5 atoms, this speak the form in the same time this structure and necessitaed greater energy. In Fig. 7 the cation cluster of the Fig. 3c) VI are greater values $1.57 \mathrm{eV}$. For the anion and cation, Fig. 7, the values are very near.

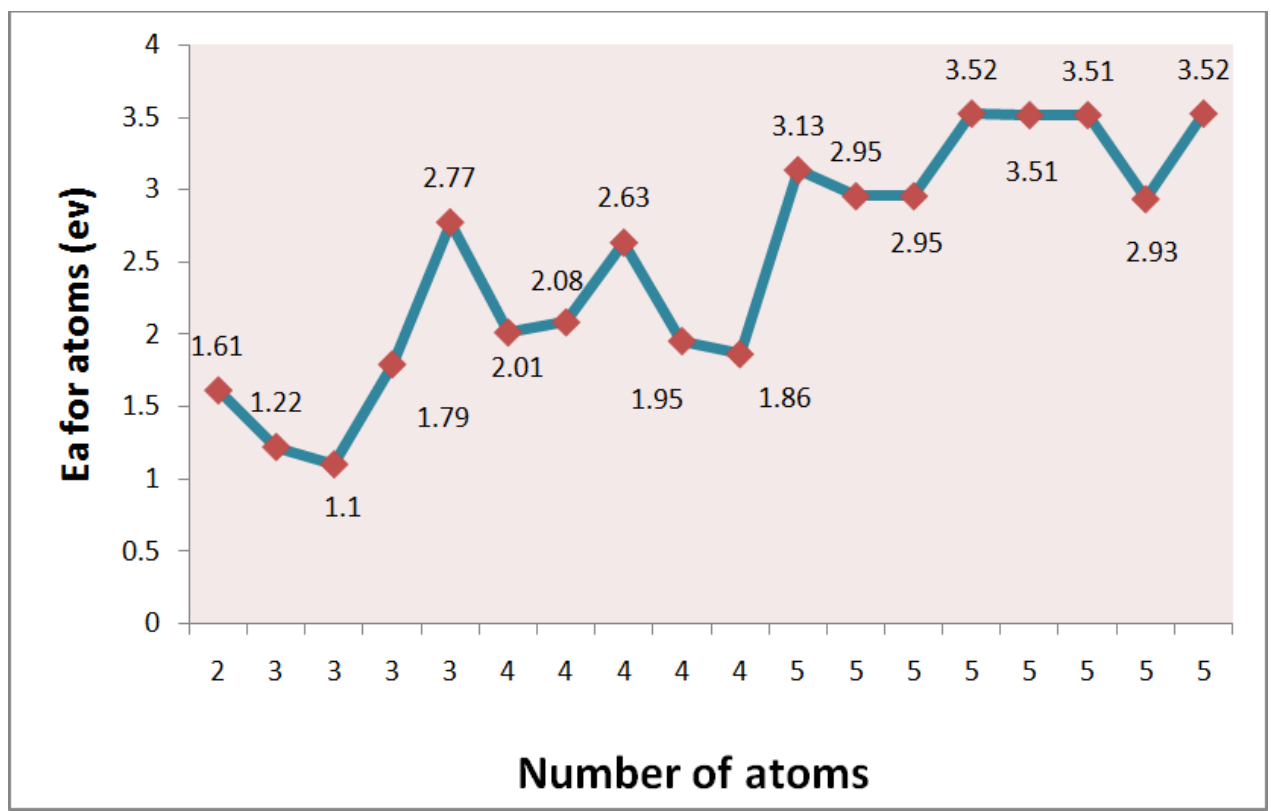

Fig. 5. Biding energy for atom for neutral clusters. The cluster for 2 atoms are: Fig 1 a; for 3 atoms: Fig. 2 a)I, a)II, a)III, a)IV, and a)V; for 4 atoms are: Fig. 3 a)I, a)II, a)III, a)IV, and a) V; for 5 atoms are: Fig. 4 a)I, a)II, a)III, a)IV, a)V, a)VI, a)IX, and a)X. The values are in the figure.

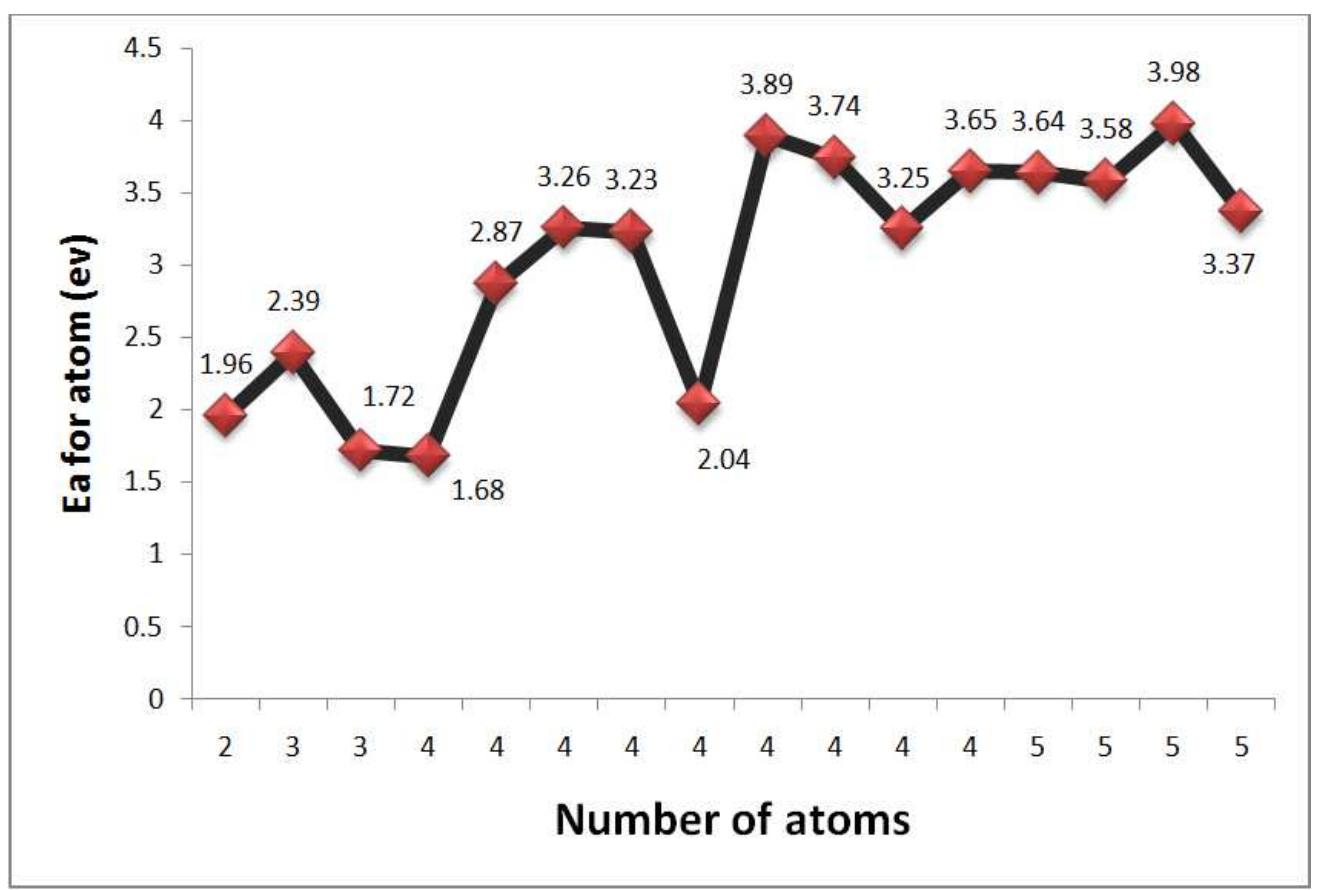

Fig. 6. Biding energy for atom for anion clusters. The clusters for 2 atoms are: Fig. 1 b), for 3 atoms are: Fig. 2 b)I, b)II, and b)III; for 4 atoms are: Fig. 3 b)I, b)II, b)III, b)IV, b)V, b)VI, b)VII, b)VIII, and b)IX, The clusters for 5 atoms are: Fig. 4 b)I, b)II, b)III, and b)IV. The values are in the figure. 


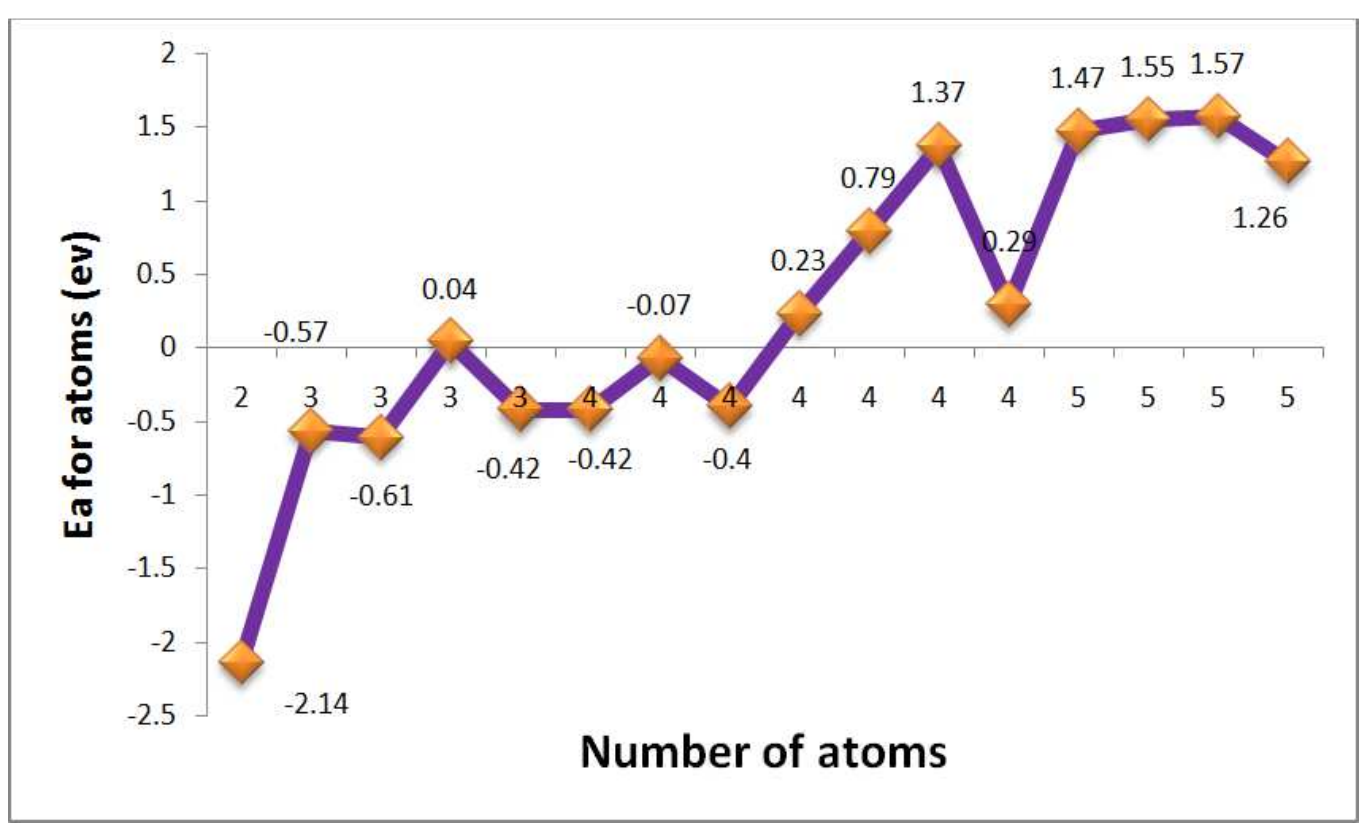

Fig. 7. Biding energy for atom for cation clusters. The clusters for 2 atoms are: Fig. 1 c); for 3 atoms are: Fig. 2 c)I, c)II, c)III, and c)IV; for 4 atoms are: Fig. 3 c)I, c)II, c)III, c)IV, c)V, c)VI, and c)VII; for 5 atoms are: Fig. 4 c)I, c)II, c)III, c)IV and c)V. The values are in the figure.

\section{Ionization Potential and Electronic Affinity}

\subsection{Vertical and Adiabatic Electronic Affinity}

The calculus for electronic affinites has been calculated with $A=E_{n}-E_{n}^{-}$, where $E_{n}$ is the total energy for neutral species optimized and $E_{n}^{-}$is the total energy for the anion species after optimized. For the vertical electronic affinities are calculated with the neutral form optimized and single point for anion and cation cluster.

The value -1.14 in Fig. 8 for cluster with 3 atoms speak that this cluster are not stable. For 5 atoms are need vergy large value $4.07,4.26 \mathrm{eV}$, Fig. 8 .

In Fig. 9 for structure with 5 atoms the vertical electronic afinity are little, the very large value are for Fig. 4 a) III, 3.11 $\mathrm{eV}$. The tendency and values for adiabatic and vertical electon affinity are different. Are not experimental date. For the vertical electronic afinity are variation odd even in the cluster.

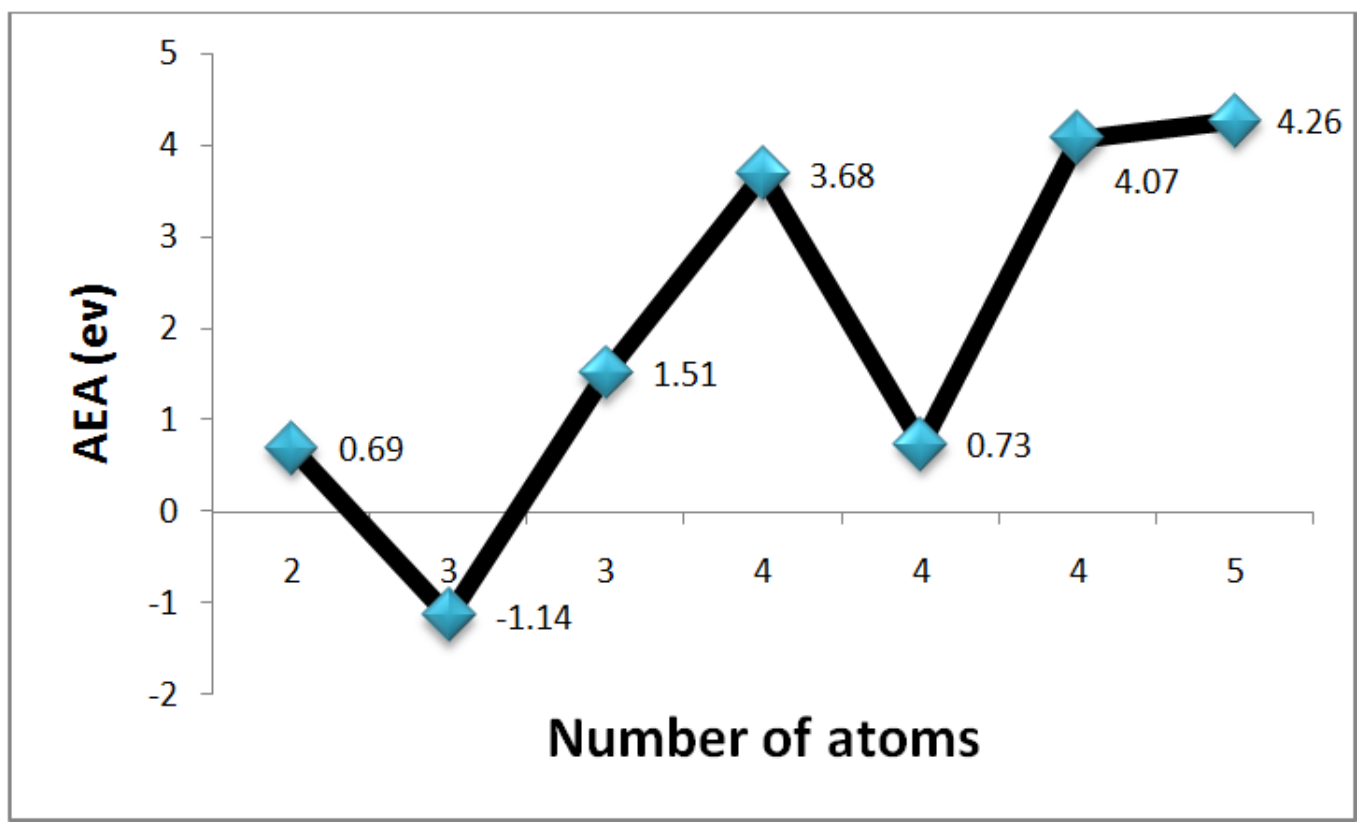

Fig. 8. Adiabatic electronic afinity for silver cluster employed. Vs. Number of atoms in the cluster. 


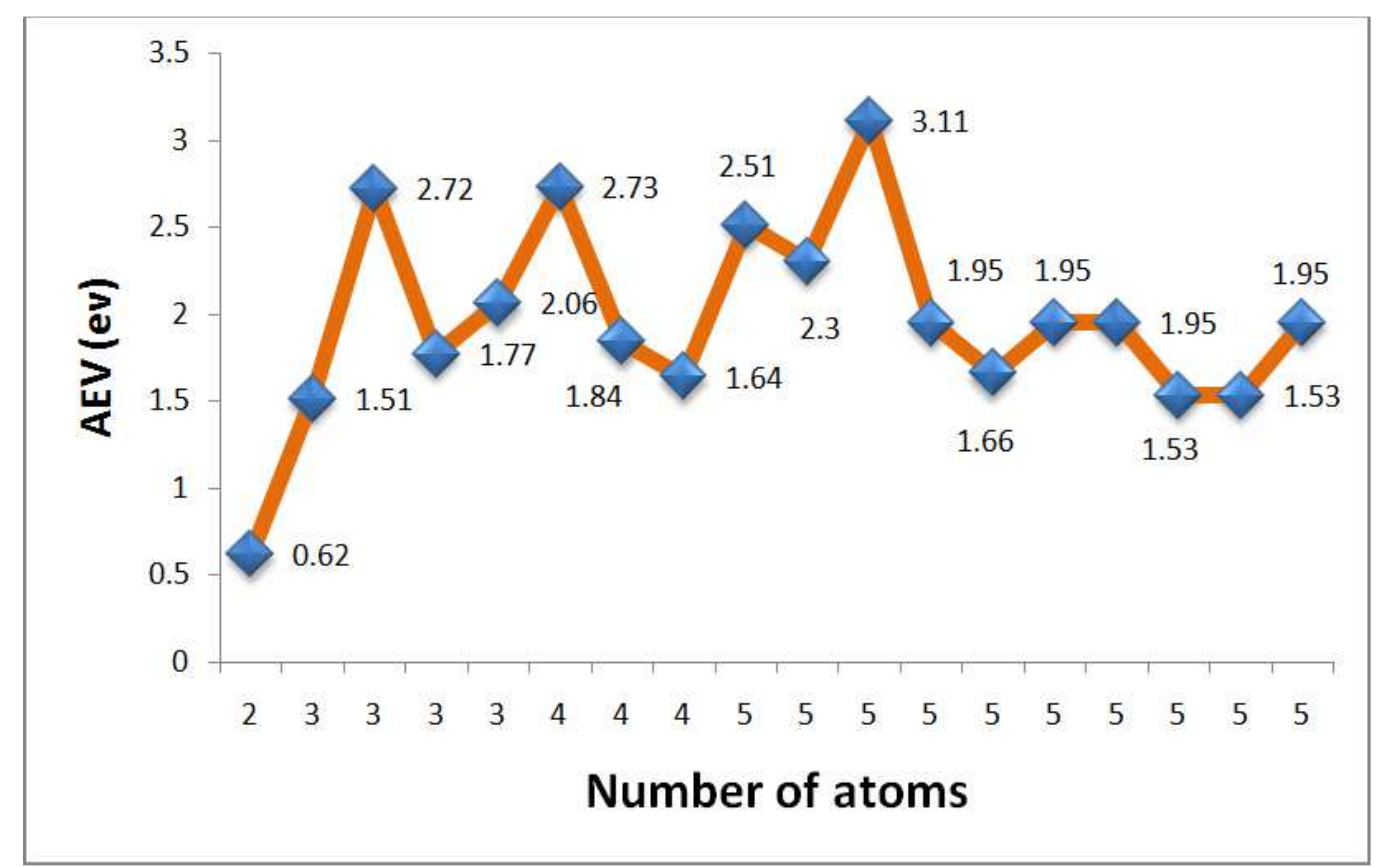

Fig. 9. Vertical electronic afinity for silver cluster employed DFT. VS. Number of atoms in the cluster.

\subsection{Vertical and Adiabatic Ionization Potential}

The adiabatic ionization potential are defined how the energy of transition in origin between the basal state of cation and the basal state of neutral with $I=E_{n}^{+}-E_{n}$, where $E_{n}^{+}$is the total energy of species of cations after optimization the structure and $E_{n}$ is the total species neutral optimizated. For vertical ionization potential are empoyed the neutral values for the structure optimized and for the cation sigle point with the neutral structure.

In Fig. 10 are view the mas greater value ar for cluster with 5 atoms, $10.27 \mathrm{eV}$ and for 3 atoms are $10.17 \mathrm{eV}$. In figure 11 are see the cluster with 5 atoms are very near value with compared with the another structure. Are not experimental data.

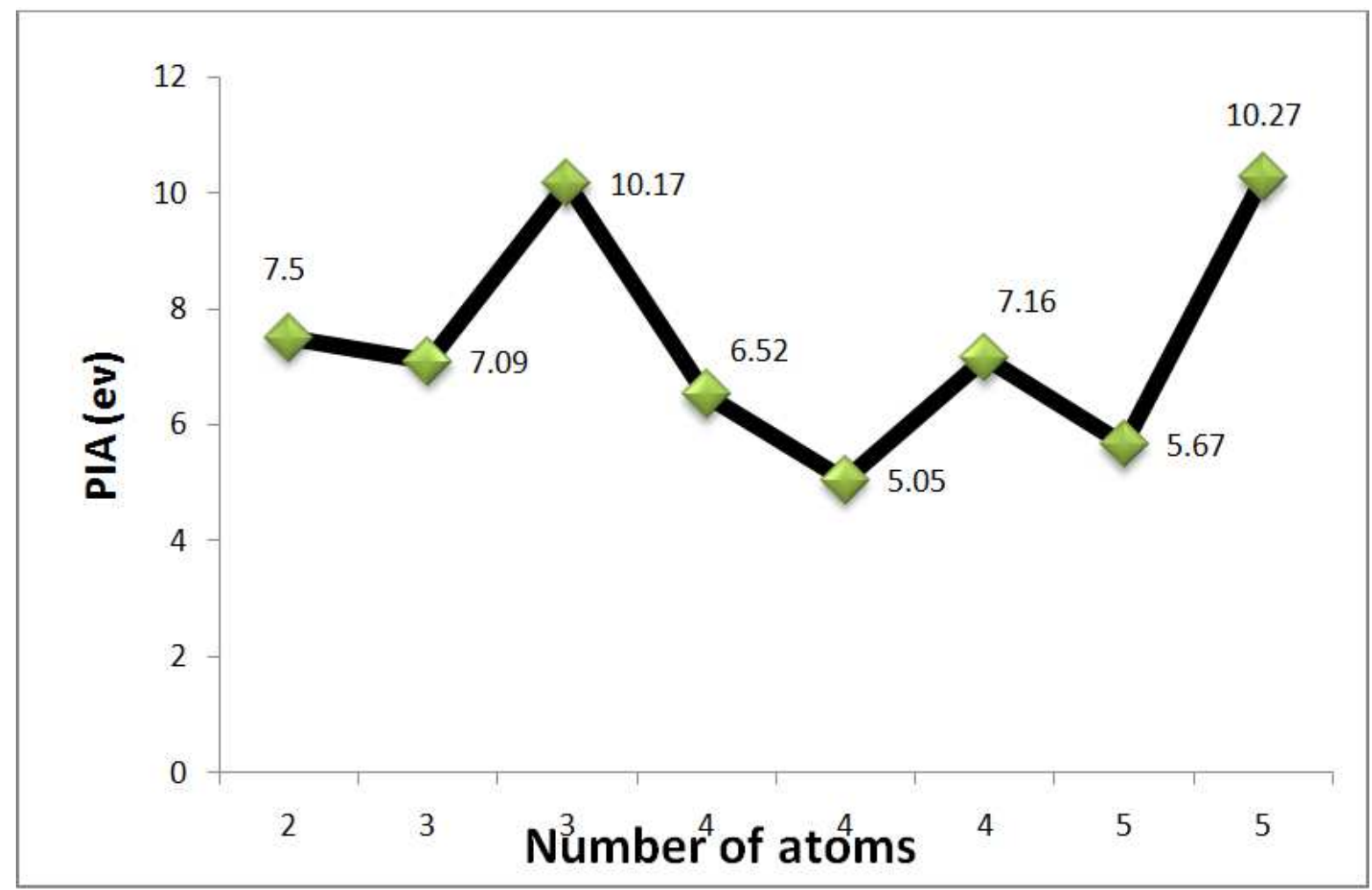

Fig. 10. Adiabatic electronic potential for silver cluster employed HF. Vs. Number of atoms in the cluster. 


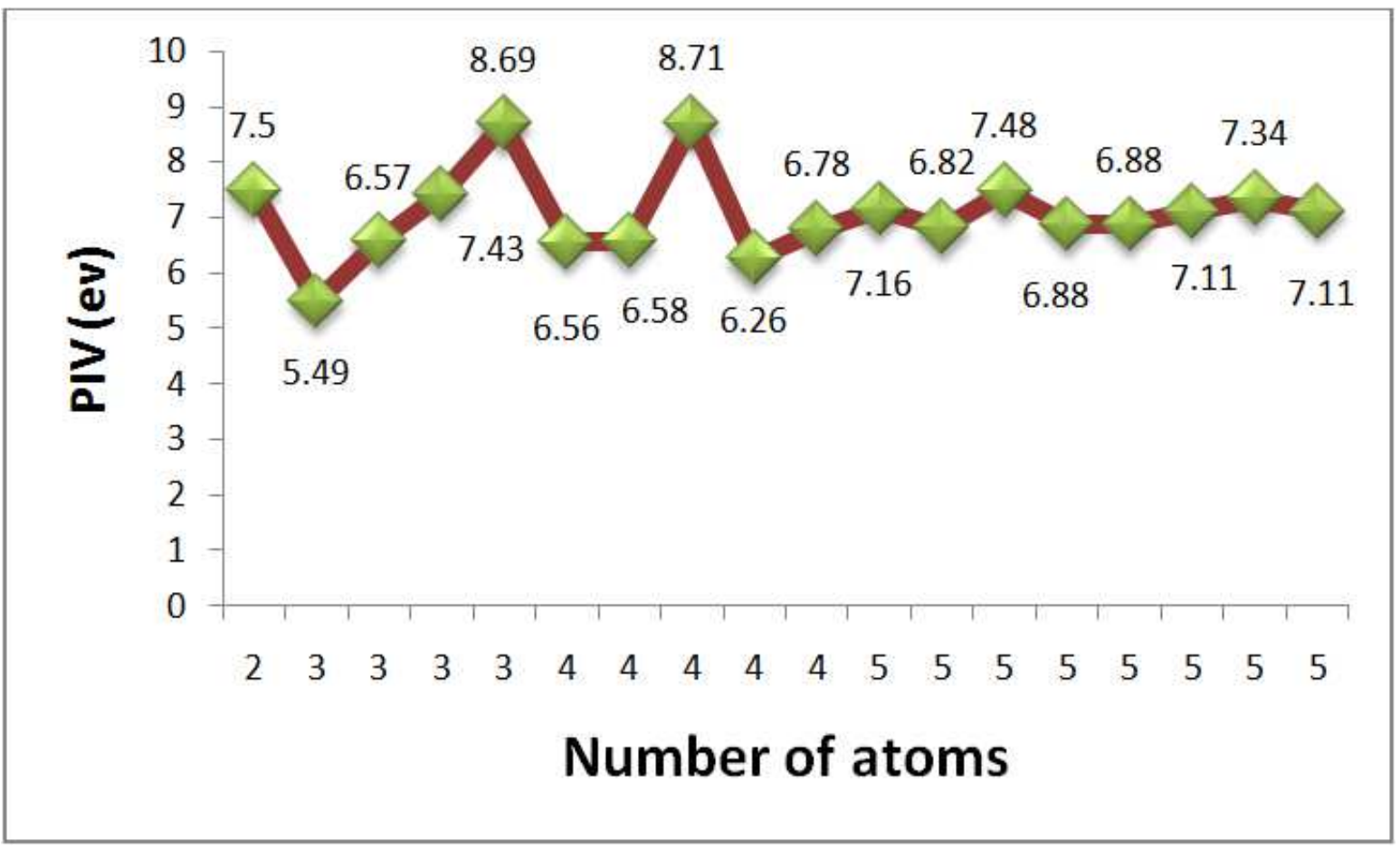

Fig. 11. Vertical ionization potential for silver cluster employed HF. Vs. Number of atoms in the cluster.

\section{Frecuencies}

The Table 1-9 are show the values of frecuencies for all structure. Are not experimental data for this clusters.

Tabla 1. The armonic frecuencia (in cm-1) of the more stable cluster with 2 atoms.

\begin{tabular}{lll}
\hline (3) ${ }^{2.56}(3)$ & $(3)^{2.43}-3$ & (3) \\
\hline 289.4678 & 277.5002 & 296.4516 \\
\hline
\end{tabular}

Tabla 2. The armonic frecuencia (in cm-1) of the more stable neutral cluster with 3 atoms.

\begin{tabular}{|c|c|c|c|}
\hline (3) $0^{2.38}$ & (3) 2.59 & (3) & \\
\hline 61.2540 & 60.2707 & 61.8344 & 176.3551 \\
\hline 61.2541 & 60.2708 & 248.7972 & 217.6540 \\
\hline 170.2134 & 169.3103 & 310.9345 & 515.8439 \\
\hline
\end{tabular}

Tabla 3. The armonic frecuencia (in $\mathrm{cm}$-1) of the more stable neutral cluster with 4 atoms.

\begin{tabular}{|c|c|c|c|c|}
\hline $\int_{2.46}^{2.46}$ & $a_{2}$ & $\int_{2.43}^{2.47}$ & 2.68 & 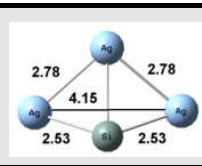 \\
\hline 38.0908 & 77.1104 & 17.8624 & 59.1809 & 39.1314 \\
\hline 50.6923 & 89.2589 & 40.3779 & 86.6282 & 114.5355 \\
\hline 164.9246 & 195.5846 & 95.1723 & 121.3310 & 120.2875 \\
\hline 247.1882 & 220.0214 & 176.0888 & 170.4721 & 124.9824 \\
\hline 293.9536 & 254.1555 & 265.6528 & 202.6184 & 265.6886 \\
\hline 302.3129 & 297.3688 & 481.6978 & 270.0633 & 277.5728 \\
\hline
\end{tabular}


Tabla 4. The armonic frecuencia (in cm-1) of the more stable neutral cluster with 5 atoms.

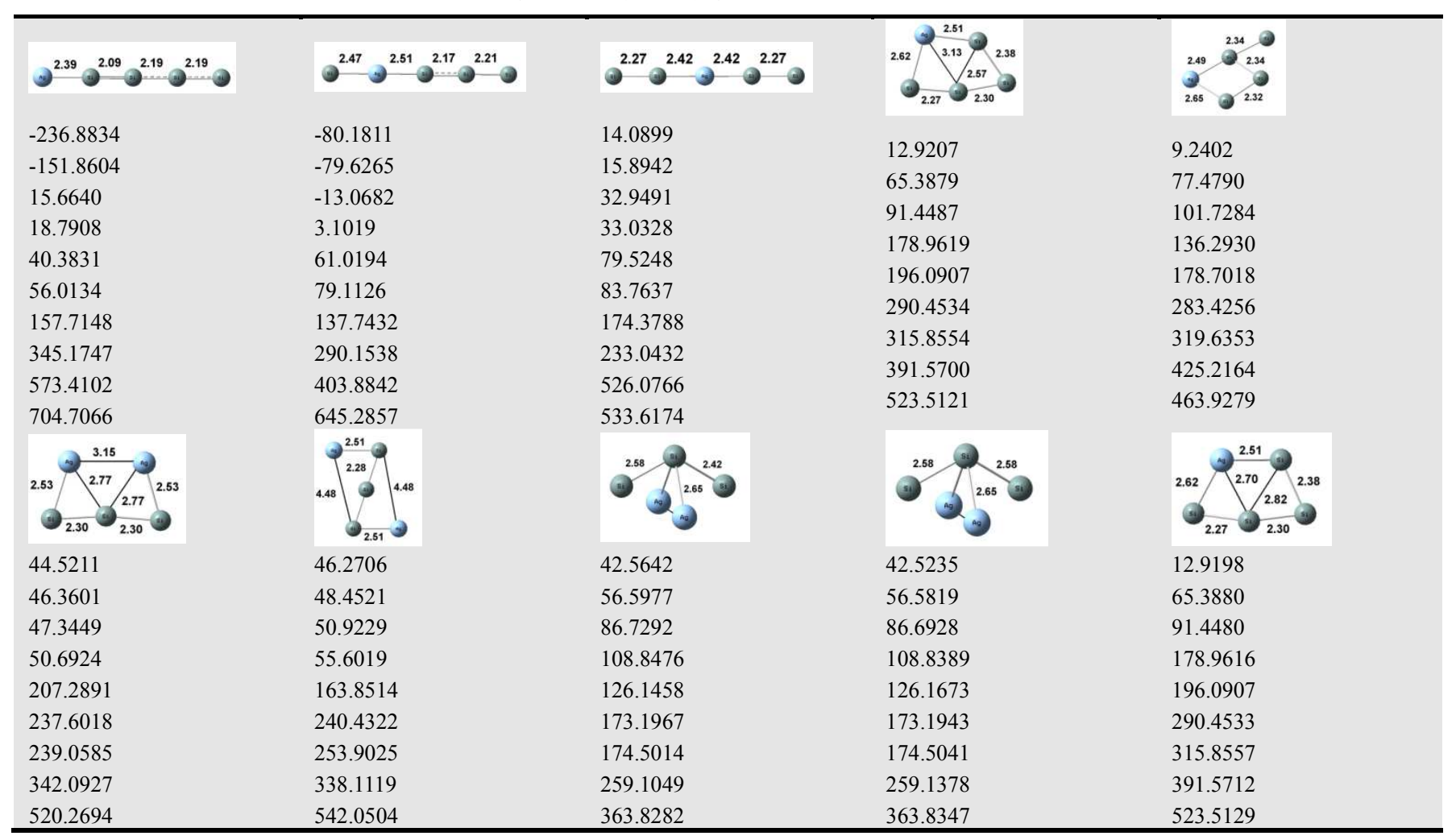

Tabla 5. The armonic frecuencia (in cm-1) of the more stable anion cluster with 3 atoms.

\begin{tabular}{|c|c|c|}
\hline 2.49 & 4.22 & \\
\hline $\begin{array}{l}66.5929 \\
224.6393 \\
294.4007\end{array}$ & $\begin{array}{l}79.0991 \\
291.0727 \\
297.0563\end{array}$ & $\begin{array}{l}142.5894 \\
149.8741 \\
173.4504 \\
428.1234\end{array}$ \\
\hline
\end{tabular}

Tabla 6. The armonic frecuencia (in cm-1) of the more stable anion cluster with 4 atoms.

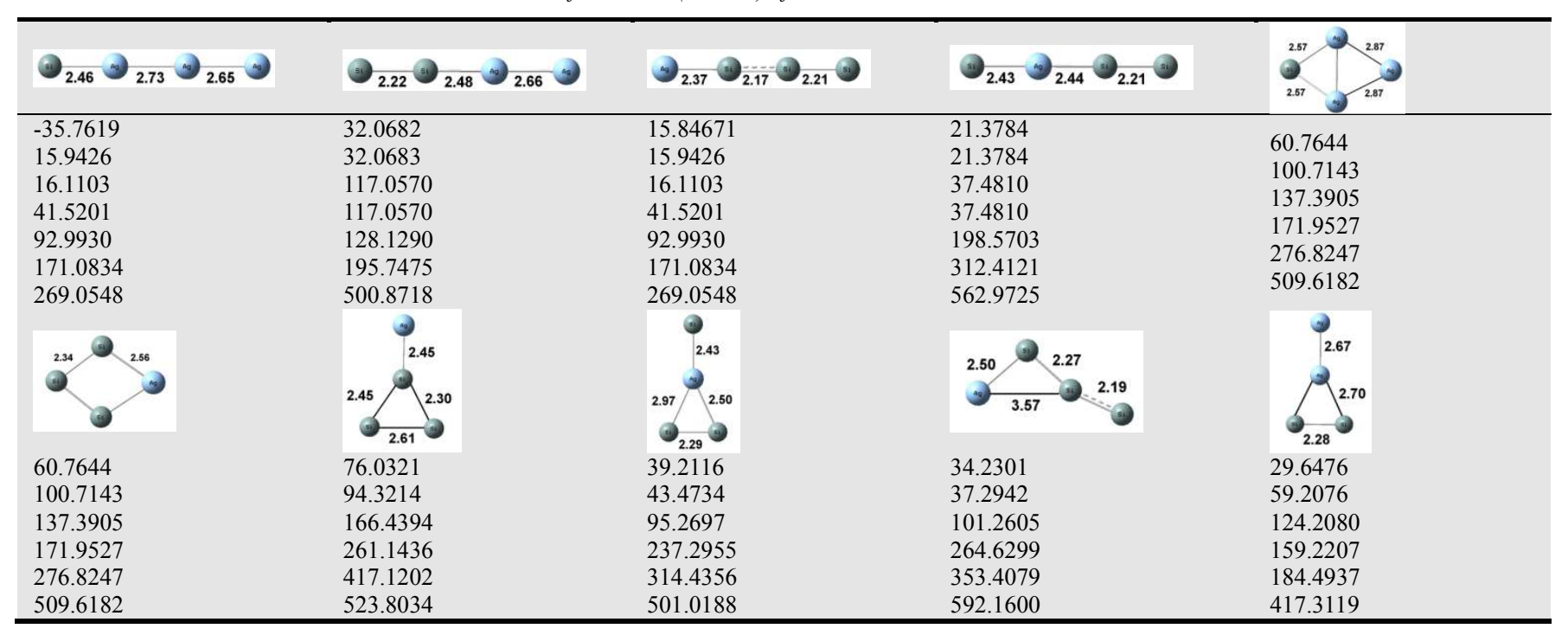


Tabla 7. The armonic frecuencia (in cm-1) of the more stable anion cluster with 5 atoms.

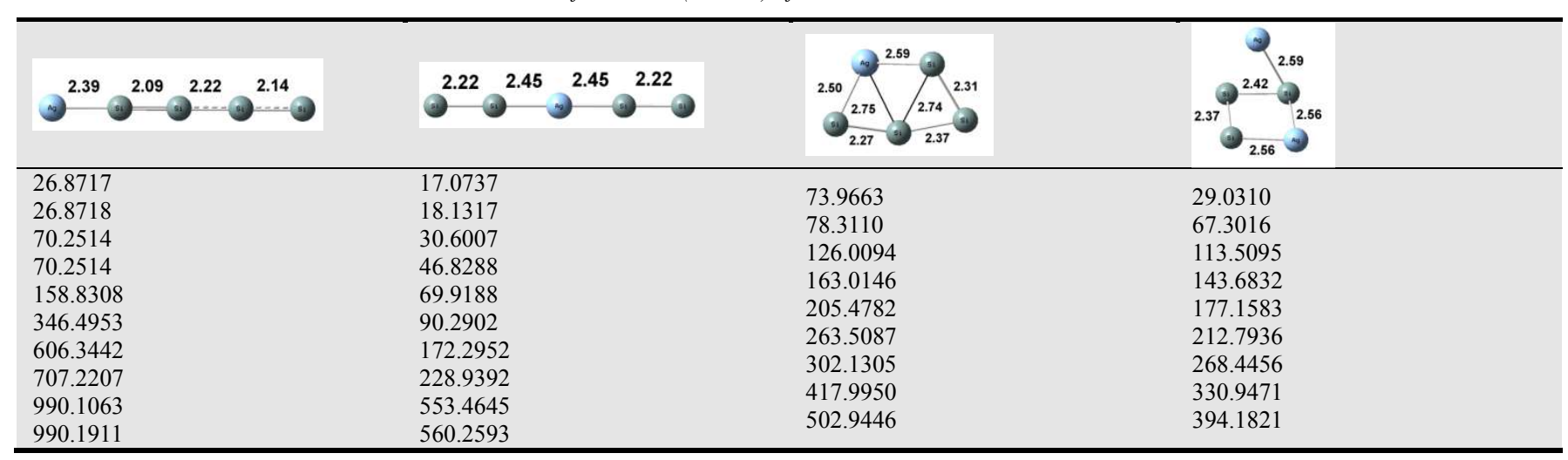

Tabla 8. The armonic frecuencia (in cm-1) of the more stable cation cluster with 3 atoms.

\begin{tabular}{|c|c|c|c|}
\hline 4.58 & 2.72 & 4.46 & $\int_{2.52}^{2.68}$ \\
\hline 45.9334 & 133.0302 & 74.3294 & 142.2687 \\
\hline 232.0150 & 142.8373 & 279.4977 & 198.9206 \\
\hline 314.5689 & 250.5726 & 297.7459 & 361.7706 \\
\hline
\end{tabular}

Tabla 9. The armonic frecuencia (in cm-1) of the more stable cation cluster with 4 atoms.

\begin{tabular}{|c|c|c|c|}
\hline$\overbrace{2.46} \underbrace{}_{2.72}$ & $\sigma_{2.63} \bigcirc_{2.50} \Im_{2.58}$ & $\int_{2.48} \bigcirc_{2.48}$ & $\int_{2.55}^{2.55}$ \\
\hline-37.2755 & -56.3408 & 21.3554 & 42.8461 \\
\hline-37.2752 & 19.4096 & 21.3554 & 84.9234 \\
\hline 35.1106 & 33.1875 & 90.3077 & 109.0936 \\
\hline 35.1284 & 119.7142 & 130.6749 & 126.8424 \\
\hline 90.8406 & 204.2132 & 130.6749 & 242.1449 \\
\hline 167.8173 & 252.7979 & 196.0670 & 279.4739 \\
\hline 290.7447 & 299.6463 & 336.3791 & \\
\hline $2.62 \int_{2.84}^{2.64}$ & 2.52 & $\int_{2.50}^{0.74}$ & \\
\hline 88.5063 & 29.7044 & 46.2545 & \\
\hline 93.1119 & 35.5871 & 81.9702 & \\
\hline 126.9542 & 88.6014 & 103.8632 & \\
\hline 169.5154 & 198.3702 & 161.8707 & \\
\hline 343.8686 & 380.1266 & 169.7107 & \\
\hline 1797.5510 & 576.0177 & 267.2918 & \\
\hline
\end{tabular}

Tabla 10. The armonic frecuencia (in cm-1) of the more stable cation cluster with 5 atoms.

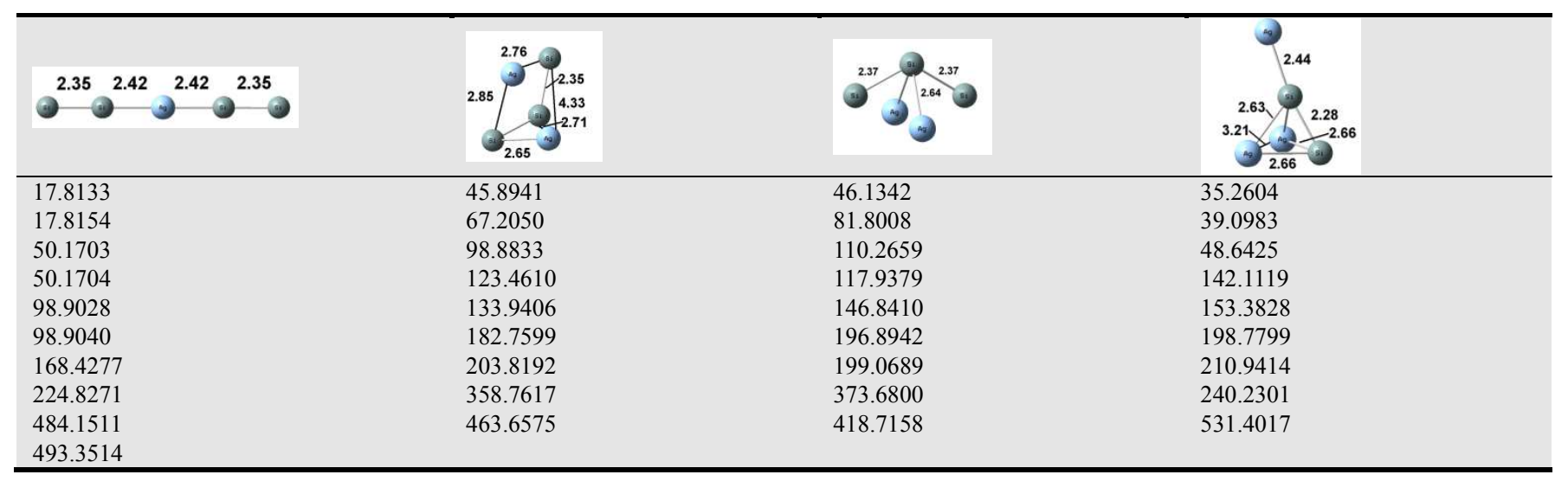




\section{Hardness}

Are calculated hardness with the objective see the reactivity of the cluster taken in account the relativistic effects and electronic correlation and see what silver cluster digest how soft acid or soft base or hard acid or hard base. The hardness are calculated with $\eta=(P I-A E) / 2$, where PI are the adiabatic ionization potential and $\mathrm{AE}$ are the adiabatic electron affinity.

In the Table 13-15 are the value obtained for the difference HOMO-LUMO (gap) for the clusters employed the Klopman theorie [6].

In Fig 12 the cluster with 3 atoms are hard and with 5 atoms are soft. This value a speak the energy for acepted electron in cluster. The tendencies in HOMO-LUMO are different for anion, cation and neutral cluster.

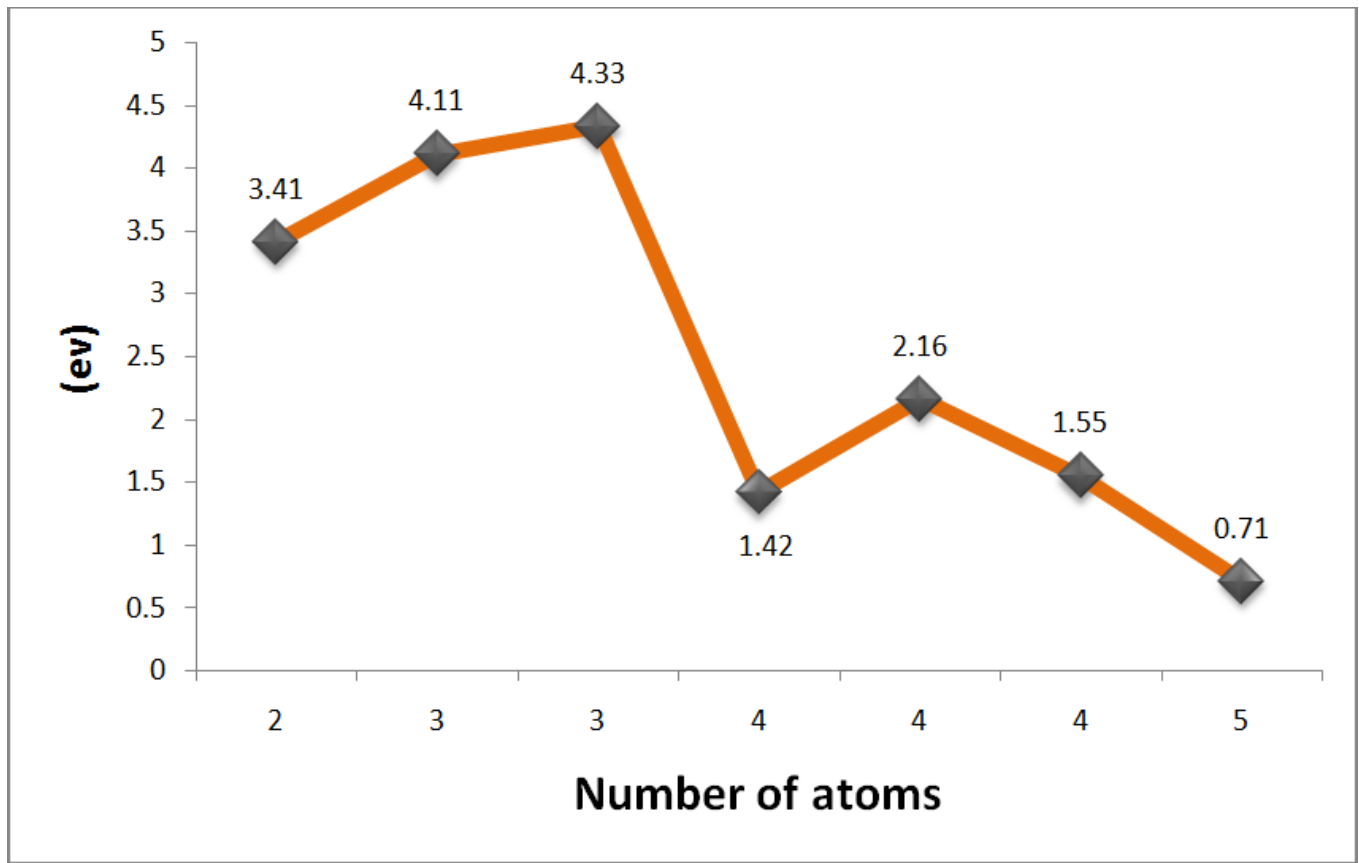

Fig. 12. Hardness ( $\eta$ ) for $A g_{x} S i_{y}$ cluster. Vs. Number of the atoms in the cluster.

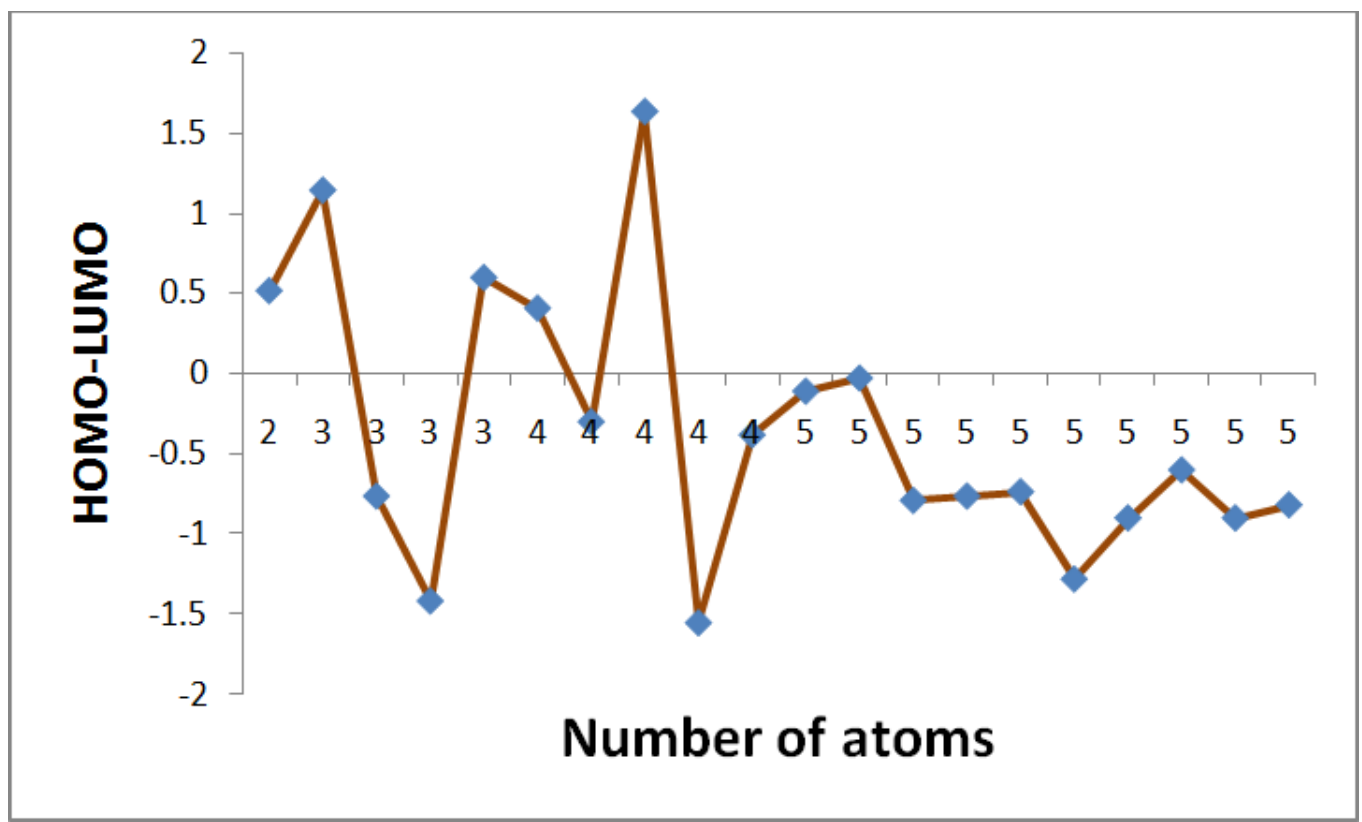

Fig. 13. HOMO-LUMO for $A g_{x} S_{i_{y}}$ neutral cluster. Vs. Number of the atoms in the cluster. 


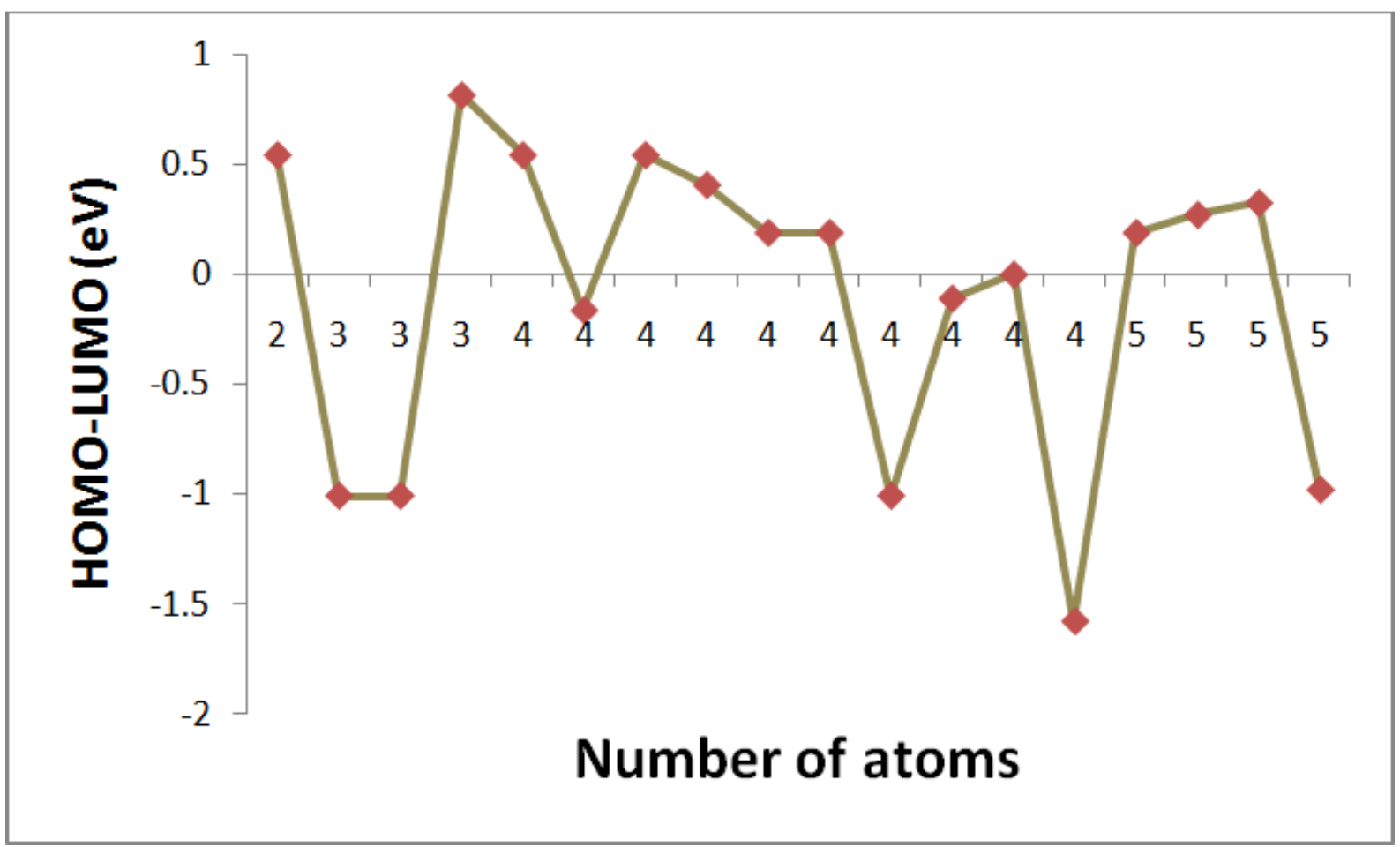

Fig. 14. HOMO-LUMO for Ag $\mathrm{Si}_{\mathrm{y}}$ anion cluster. Vs. Number of the atoms in the cluster.

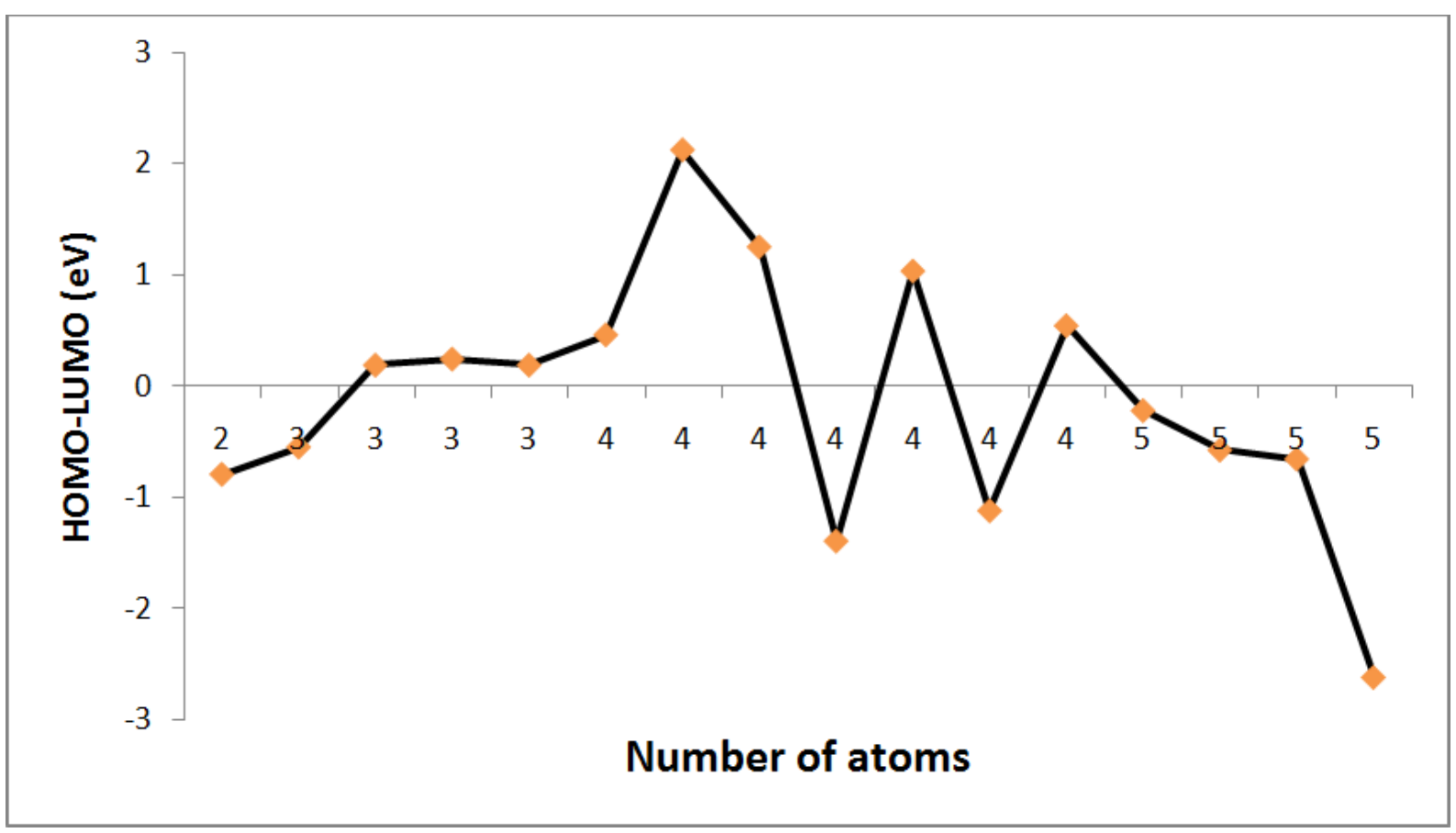

Fig. 15. HOMO-LUMO for $\mathrm{Ag}_{x} \mathrm{Si}_{y}$ anion cluster. Vs. Number of the atoms in the cluster.

\section{Enthalpies and Free Energy}

The enthalpies and free energy, are speak of the tip of reaction endothermic and exothermic when this cluster are reactioned with another structure, Fig. 16-21. The value are near of 100-3000 Kcal/mol. The tendency for free energy and enthalpies are the sam for anion, cation and neutral cluster.
Very stable are the neutral structure in Fig. 4a) VIII and IX with near of $-3000 \mathrm{kcal} / \mathrm{mol}$ energy and piramidal structure for cluster with 4 atoms, Fig.16. In anion cluster with linear estructure are more stable, Fig. 17. For estructure rombic and linear in cation cluster are very stable with the trapezoidal form for structure with 5 atoms, Fig. 20. 


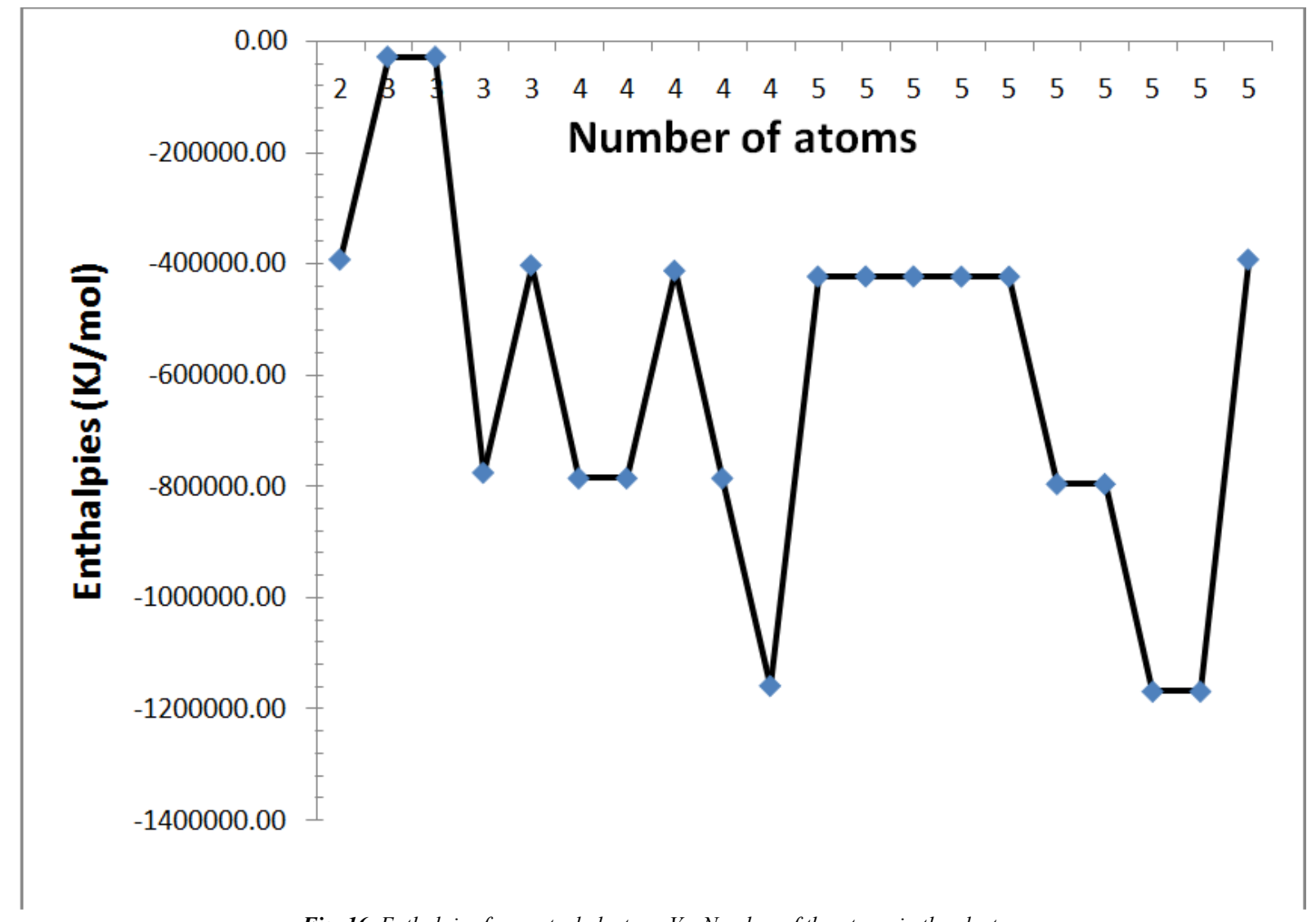

Fig. 16. Enthalpies for neutral clusters. VS. Number of the atoms in the cluster.

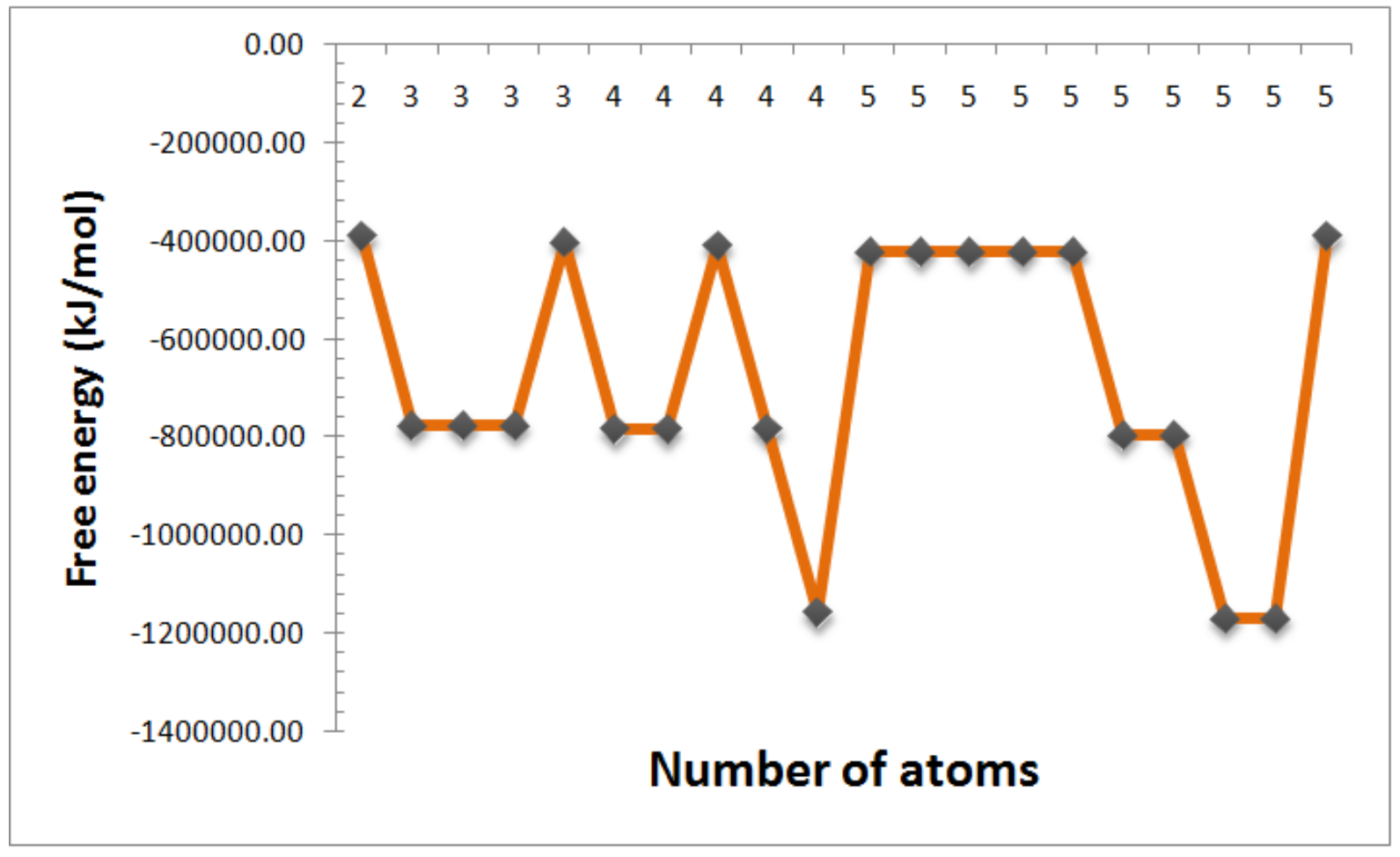

Fig. 17. Free energy for neutral clusters. Vs. Number of the atoms in the cluster. 


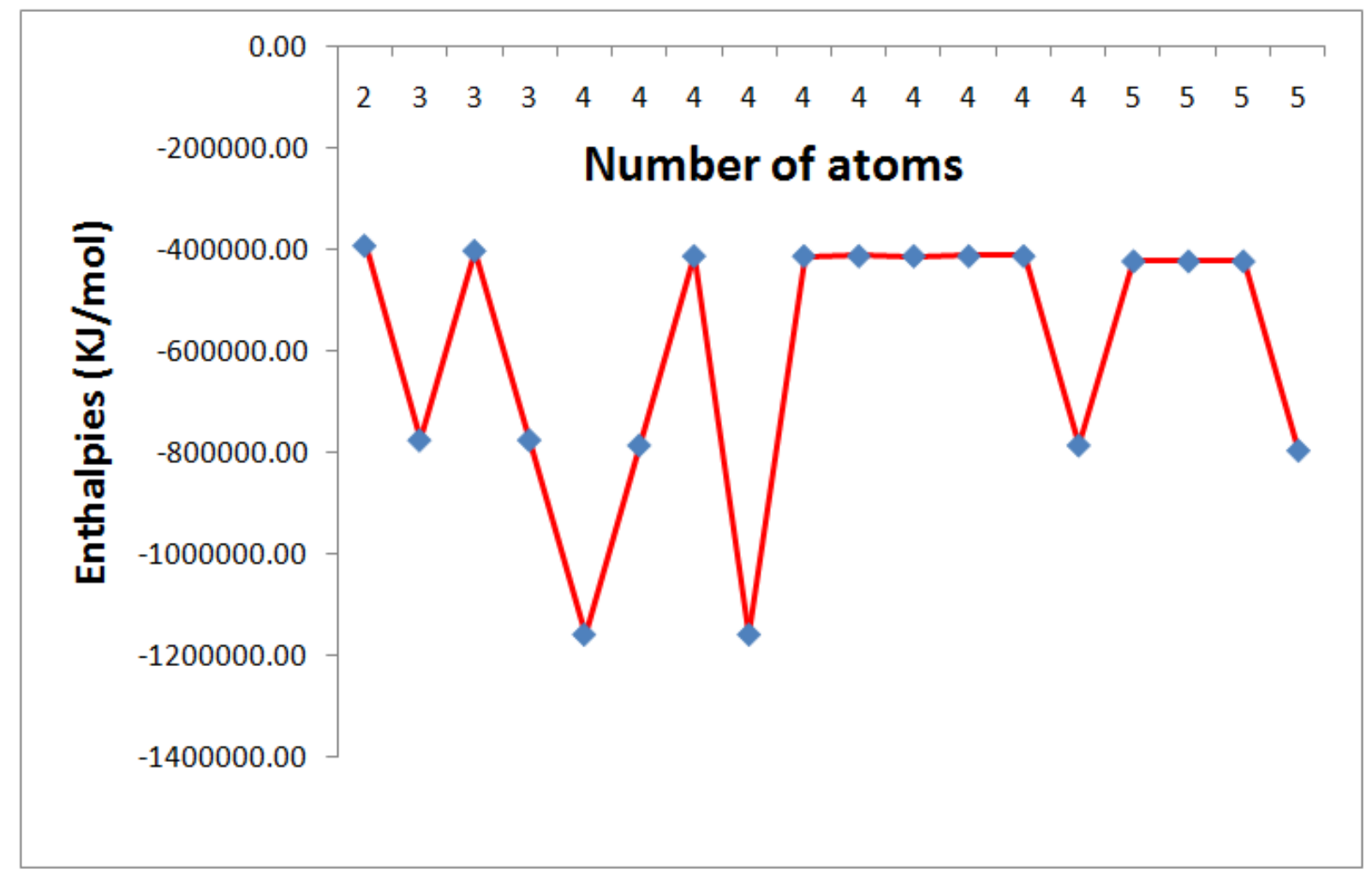

Fig. 18. Enthalpies for anion clusters. Vs. Number of the atoms in the cluster.

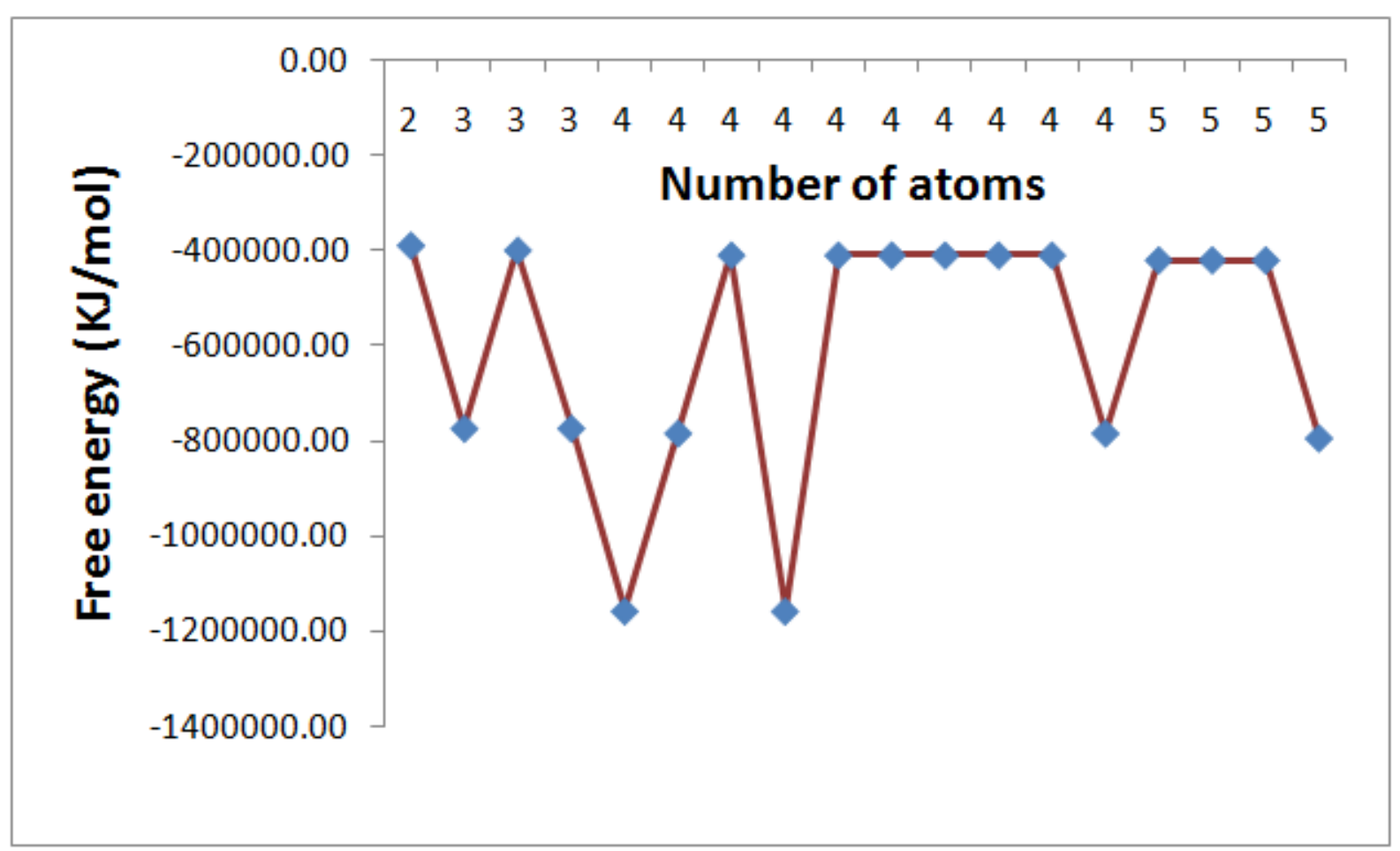

Fig. 19. Free energy for anion clusters. Vs. Number of the atoms in the cluster. 


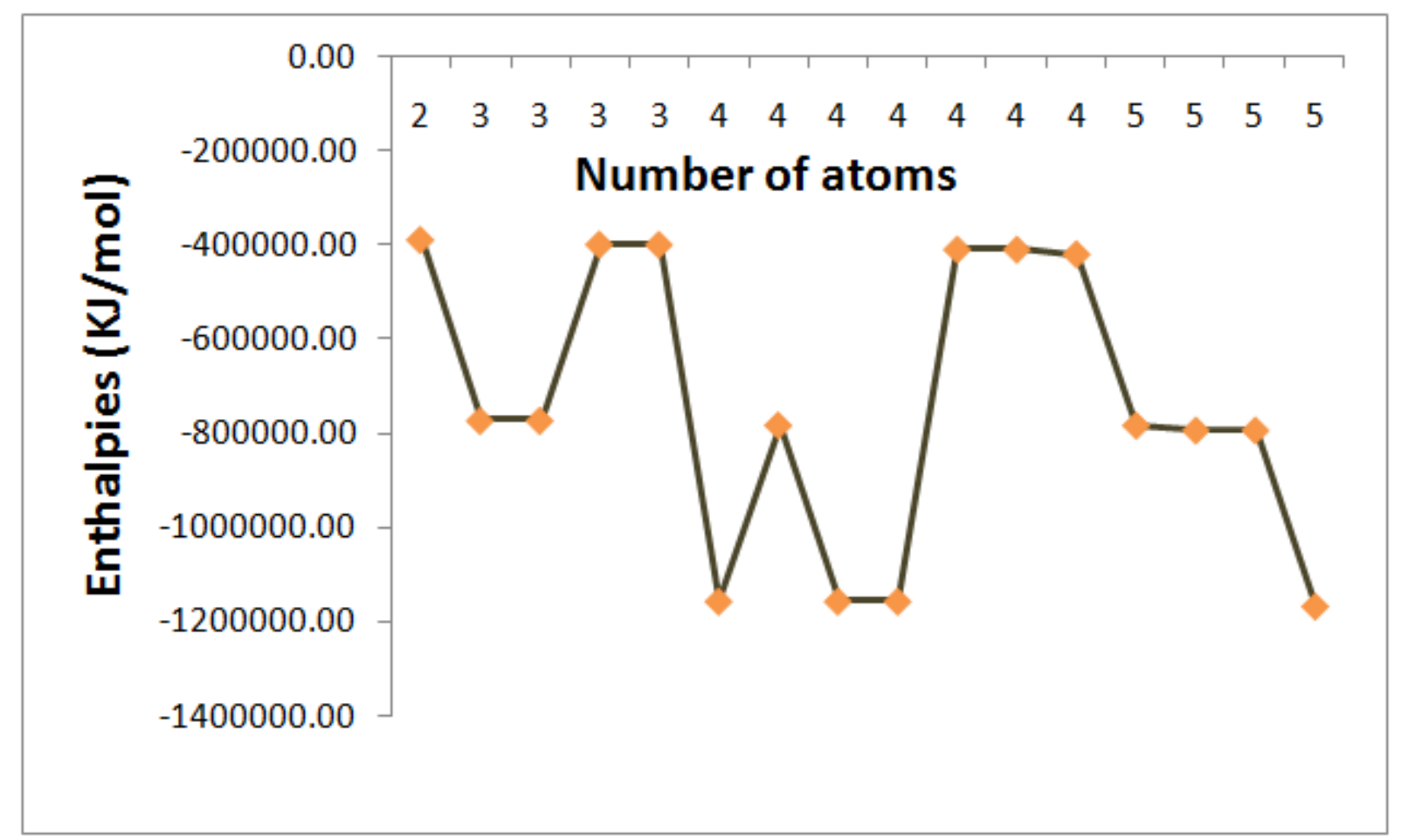

Fig. 20. Enthalpies for cation clusters. Vs. Number of the atoms in the cluster.

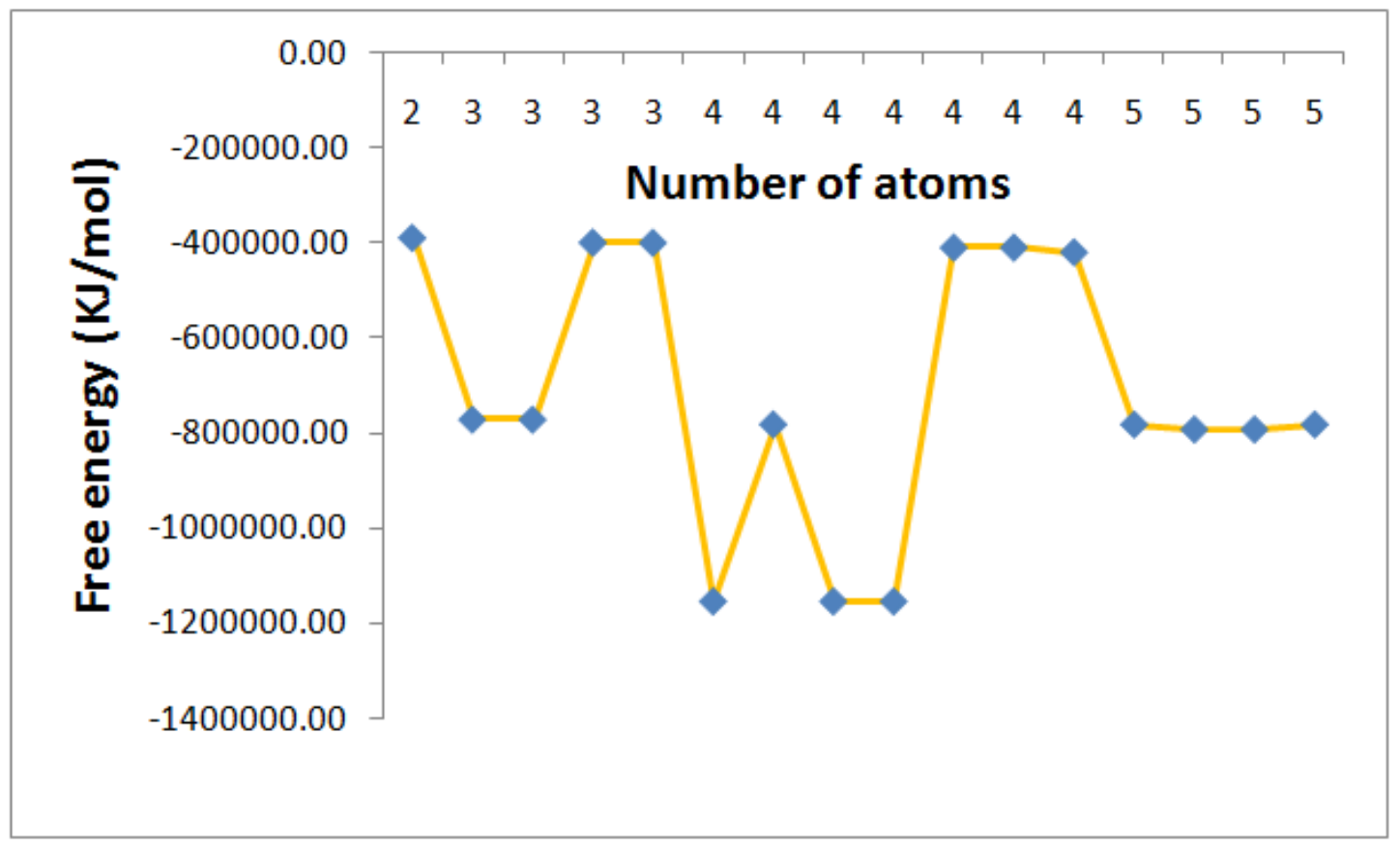

Fig. 21. Free energy for cation clusters. Vs. Number of the atoms in the cluster.

\section{Conclusions}

In this work are studie the cluster $\mathrm{Ag}_{\mathrm{x}} \mathrm{Si}_{\mathrm{y}}$ structure and their proprieties employed the PBE/LANLEDZ level of theorie with relarivistic effect. For neutral trimer cluster $\mathrm{C} 2$, A1 structure isoscele are more stable, Fig. 2 a)III. In case of neutral cluster with 4 atoms the piramidal structure are very stable. The Fig. 4 a)VII are very stable neutral cluster. For anion the linear structure are very stable, Fig. 2 b) III. In Fig.
$5 \mathrm{~b}) 4$ the anion structure are very stable with $-303.18 \mathrm{eV}$.

All $\alpha$ HOMO orbital are occuped with one electron and HOMO and LUMO with 2 electron.

Are studie the frecuencies, electronic affinity, electronic potential, binding energy for atom, enthalpies and free energy. The structure with negative frecuencies are sadlle point in PES.

The electronic affinity and electronic potential speek of the energy for accepted and grant electrons. 
The values reported for enthalpies and free energy are normal values.

All structure are soft and are reactive. Polarization is easiest when the energy gap is small for each molecule.

\section{References}

[1] G. Ortiz and P. Ballone, Phys. Rev., B 43, 1991, 6376; 44, 1991, pp. 5881.
[2] G. Ortiz, Phys. Rev., B 45, 11, 1992, pp. 328.

[3] J. P. Perdew, K. Burke, M. Ernzerhof, Phys. Rev. Lett. 77, 1996, pp. 3865.

[4] G. B. Bacskay, Chem. Phys. , 1981, 61, pp. 385.

[5] Mariana V. Popa, International Journal of Computational and Theoretical Chemistry, 2014, 2(6), pp. 46.

[6] C.E. Moore, Atomic energy levels, NSRDS-NBS Circular No. 467, USGPO, Washington, pp. 1949. 